\title{
New derivation of redshift distance without using power expansions. II. Additional redshift distances, analyzing the data of more quasars, of some SNIa, of the one black hole in galaxy M87 as well as a newer estimation of $\mathbf{H}_{0}$
}

\author{
Steffen Haase ${ }^{* 1}$ \\ *04319 Leipzig, Germany
}

\begin{abstract}
In this part of the two-part series of essays, we first derive some equations for further physical redshift distances. We then analyze a catalog with 132,975 quasars, for which both the apparent magnitude $\mathrm{m}$ and the redshift $\mathrm{z}$ are given, in order to find the today's value of the parameter $\beta_{0}$ of the theory presented. We then use this value to determine the today's value of the radius $\mathrm{R}_{0 \mathrm{a}}$ of the Friedmann sphere using a magnitude redshift diagram of 19 SNIa.

With the help of the known values of $\mathrm{R}_{0_{\mathrm{a}}}$ and $\beta_{0}$, statements about astrophysical data from the black hole in the galaxy M87 can be made. In addition, the today's Hubble parameter $\mathrm{H}_{0}$ results from both parameters.

Furthermore, we calculate the values of the further physical redshift distances for the black hole in M87 and all 19 SNIa.

The resulting parameter values are: $\beta_{0} \approx 0.731, \mathrm{R}_{0 \mathrm{a}} \approx 2,712.48 \mathrm{Mpc}$ and $\mathrm{H}_{0} \approx 65.638 \mathrm{~km} /(\mathrm{s} \cdot \mathrm{Mpc})$. The today's mass density of the Friedmann sphere is $\rho_{0} \approx 4.843 \times 10^{-27} \mathrm{~g} / \mathrm{cm}^{3}$. For the mass of the Friedmann sphere we find $\mathrm{M}_{\mathrm{FK}} \approx 1.206 \times 10^{56} \mathrm{~g}$.

Annotation:

Knowledge of the first part [1] of the series of articles is a prerequisite for understanding this article.
\end{abstract}

Key words: relativistic astrophysics, theoretical and observational cosmology, redshift, Hubble parameter, quasar, black hole, SNIa, galaxy, M87

\section{PACS NO:}

1 steffen_haase@ $@$ vodafone.de

\section{Contents}

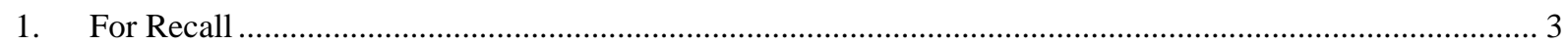

2. Derivation of further physical redshift distances ............................................................................. 3

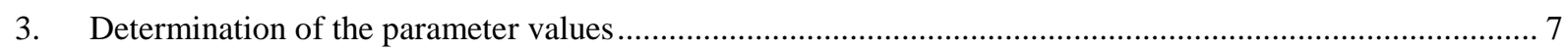

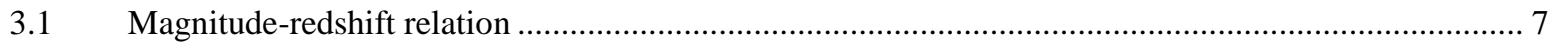

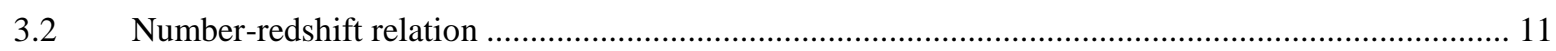

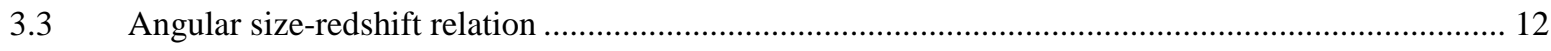

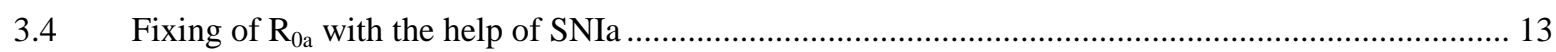

3.5 Calculation of the further redshift distances for the SNIa and M87 ............................................... 15

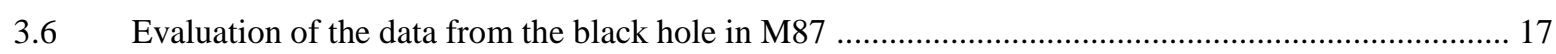

3.7 Maximum values known today: Galaxy UDFj-39546284 and Quasar J0313 .................................... 19

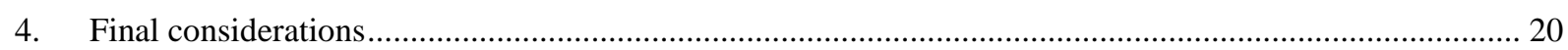

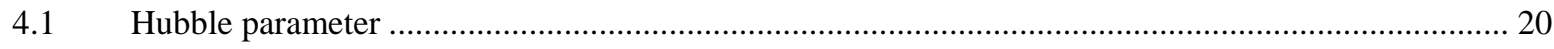

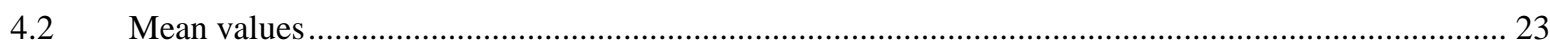

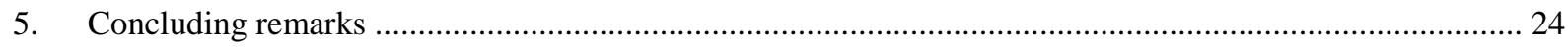

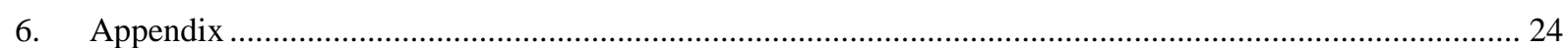




\section{List of Figures}

Figure 1. Redshift distance Rea normalized to the distance R0a

Figure 2. Redshift distance R0e normalized to the distance R0a for various values of the parameter $\beta 0$

Figure 3. Redshift distance Ree normalized to the distance R0a for different values of the parameter $\beta 0$

Figure 4. Today's ( $\mathrm{t} 0$ ) redshift distance D0 normalized to the distance R0a for various values of the parameter $\beta 0$

Figure 5. The at that time (te) redshift distance De normalized to the distance R0a for various values of the parameter $\beta 0$

Figure 6. Magnitude-redshift diagram for all 132,975 quasars according to M.-P. Véron-Cetty et al. [2].

Figure 7. Magnitude-redshift diagram of the mean values <zi $>$ and $<\mathrm{mi}>$ with inserted standard deviations $\sigma \mathrm{m}_{\text {, }}$ and $\sigma \mathrm{Z}_{\mathrm{i}}$

Figure 8. Magnitude-redshift diagram for 132,975 quasars according to M.-P. Véron-Cetty et al. [2]

Figure 9. Standard deviations $\sigma \mathrm{m}_{, \mathrm{i}}$ as a function of $\langle\mathrm{zi}\rangle$

Figure 10. Number-redshift diagram for the 132,975 quasars according to M.-P. Véron-Cetty et al. [2]

Figure 11. Angular size-redshift diagram according to K. Nilsson et al. [3]

Figure 12. Magnitude-redshift diagram for 27 SNIa according to W. L. Freedman et al. [4]

Figure 13. Redshift distance D (light path) and the further redshift distances Di $(i=0, e)$ and $R j k(j=0, e ; k=e$, a) as a function of the redshift up to $z=11$

Figure 14. Visualization of the distances Di, D and Rjk with regard to M87 and observer

Figure 15. All distances Di and D for M87, J0313 and UDFj-39546284

Figure 16. Non-approximated redshift distance D compared to the linear Hubble redshift distance

\section{List of Tables}

Table 1. Redshift distance D and the further redshift distances Di and Rjk of all 27 SNIa

Table 2. Summary of data from galaxy M87 with the black hole in it

Table 3. Redshift distances Di, D and Rjk from the black hole in M87

Table 4. All calculated redshift distances Rjk, Di and D for the two cosmic objects with the maximum redshifts

Table 5. Expansion-related shifts in the distance of the quasar and the galaxy.

Table 6. Various distances R0a, ${ }_{i}$ of the 27 SNIai calculated using the distance modules $\mu \mathrm{i}$

Table 7. Mean values from the Quasar data set used according to [2]

Table 8. Numbers Ni summed up in the redshift intervals zi of the quasars according to [2]

Table 9. Summary of the data which we used from the 27 SNIa according to [4] 


\section{For Recall}

Based on the physical approach for the dynamic path of photons (light path) through the universe

$$
D=a_{0} r_{a}-a_{e} r_{e}=R_{0 a}-R_{e e}
$$

we found in the first part of the series of articles [1] for the non-approximated redshift distance

$$
D\left(z ; R_{0 a}, \beta_{0}\right)=\frac{R_{0 a}}{(1+z)}\left[\frac{1}{\beta_{0}}\left(1-\frac{1}{\sqrt{1+z}}\right)+z\right] .
$$

In the above equation, $\mathrm{R}_{0 \mathrm{a}}$ is the physical distance of any observer from a coordinate origin $(\mathrm{r}=0)$.

In this paper we designate all distances that are related to a coordinate origin with " $\mathrm{R}$ " and all differences of such distances with "D".

For the relations relevant in the context of cosmology, we found the magnitude-redshift relation in Part I of the essay series with the help of the redshift distance

$$
m\left(z ; m_{0 a}, \beta_{0}\right)=5 \log _{10}\left[\frac{1}{\beta_{0}}\left(1-\frac{1}{\sqrt{1+z}}\right)+z\right]-5 \log _{10}(1+z)+m_{0 a},
$$

the angular size-redshift relation

$$
\varphi\left(z ; \delta / R_{0 a}, \beta_{0}\right)=\frac{\delta}{R_{0 a}} \frac{(1+z)}{\left[\frac{1}{\beta_{0}}\left(1-\frac{1}{\sqrt{1+z}}\right)+z\right]},
$$

and the number-redshift relation

$$
\log _{10} N\left(z ; N_{0 a}, \beta_{0}\right)=3 \log _{10}\left[\frac{1}{\beta_{0}}\left(1-\frac{1}{\sqrt{1+z}}\right)+z\right]-3 \log _{10}(1+z)+\log _{10} N_{0 a}
$$

In the following chapter we calculate further physical redshift distances related to the coordinate origin and differences of these distances that are very interesting for cosmology.

\section{Derivation of further physical redshift distances}

The starting point for the derivation of the further redshift distances are the elementary equations

$$
\begin{aligned}
& (1+z)=\frac{a_{0}}{a_{e}} \quad(I, 20 a) \quad \text { and } \quad D=R_{0 a}-R_{e e} \quad(I, 15) \\
& \text { and } \quad(1+z)=\frac{a_{0} r_{a}}{a_{e} r_{a}}=\frac{R_{0 a}}{R_{e a}} \quad \text { and } \quad(1+z)=\frac{a_{0} r_{e}}{a_{e} r_{e}}=\frac{R_{0 e}}{R_{e e}} \text {. }
\end{aligned}
$$

This results in the following distances 


$$
\begin{aligned}
& R_{e e}=R_{0 a}-D \quad \text { and } \quad R_{e a}=\frac{R_{0 a}}{(1+z)} \\
& \text { and } \quad R_{0 e}=(1+z) R_{e e}=(1+z)\left(R_{0 a}-D\right)
\end{aligned} .
$$

$R_{e e}$ is the then distance between the galaxy emitting the light and the origin of the coordinates - at the time $t_{e}$ the light was emitted.

$\mathrm{R}_{\mathrm{ea}}$ is the distance of the observer's galaxy from the origin of the coordinates at that time.

$\mathrm{R}_{0 \mathrm{e}}$ is the today's - at time $t_{0}$, at which the light is absorbed by the observer - distance of the light-emitting galaxy from the origin of the coordinates.

$\mathrm{R}_{0 \mathrm{a}}$ is today's distance of the galaxy containing the observer from the origin of the coordinates.

These distances become concretely with equation (I, 31)

$$
R_{e e}\left(z ; R_{0 a}, \beta_{0}\right)=R_{0 a}\left\{1-\frac{1}{(1+z)}\left[\frac{1}{\beta_{0}}\left(1-\frac{1}{\sqrt{1+z}}\right)+z\right]\right\}
$$

and

$$
R_{0 e}\left(z ; R_{0 a}, \beta_{0}\right)=R_{0 a}\left[1-\frac{1}{\beta_{0}}\left(1-\frac{1}{\sqrt{1+z}}\right)\right]
$$

and of course too

$$
R_{e a}\left(z ; R_{0 a}\right)=\frac{R_{0 a}}{(1+z)}
$$

These distances from the coordinate origin result

$$
D_{e}\left(z ; R_{0 a}, \beta_{0}\right)=\frac{R_{0 a}}{\beta_{0}} \frac{\left(1-\frac{1}{\sqrt{1+z}}\right)}{(1+z)}
$$

$D_{e}$ is the then $\left(t_{e}\right)$ distance between the observed galaxy and the galaxy in which the observer is located.

Furthermore we find

$$
D_{0}\left(z ; R_{0 a}, \beta_{0}\right)=\frac{R_{0 a}}{\beta_{0}}\left(1-\frac{1}{\sqrt{1+z}}\right) .
$$

$\mathrm{D}_{0}$ is the today's distance between the two participating galaxies.

The following figures illustrate the equations for the further redshift distances, where we have normalized all distances to $\mathrm{R}_{0 \mathrm{a}}$. 


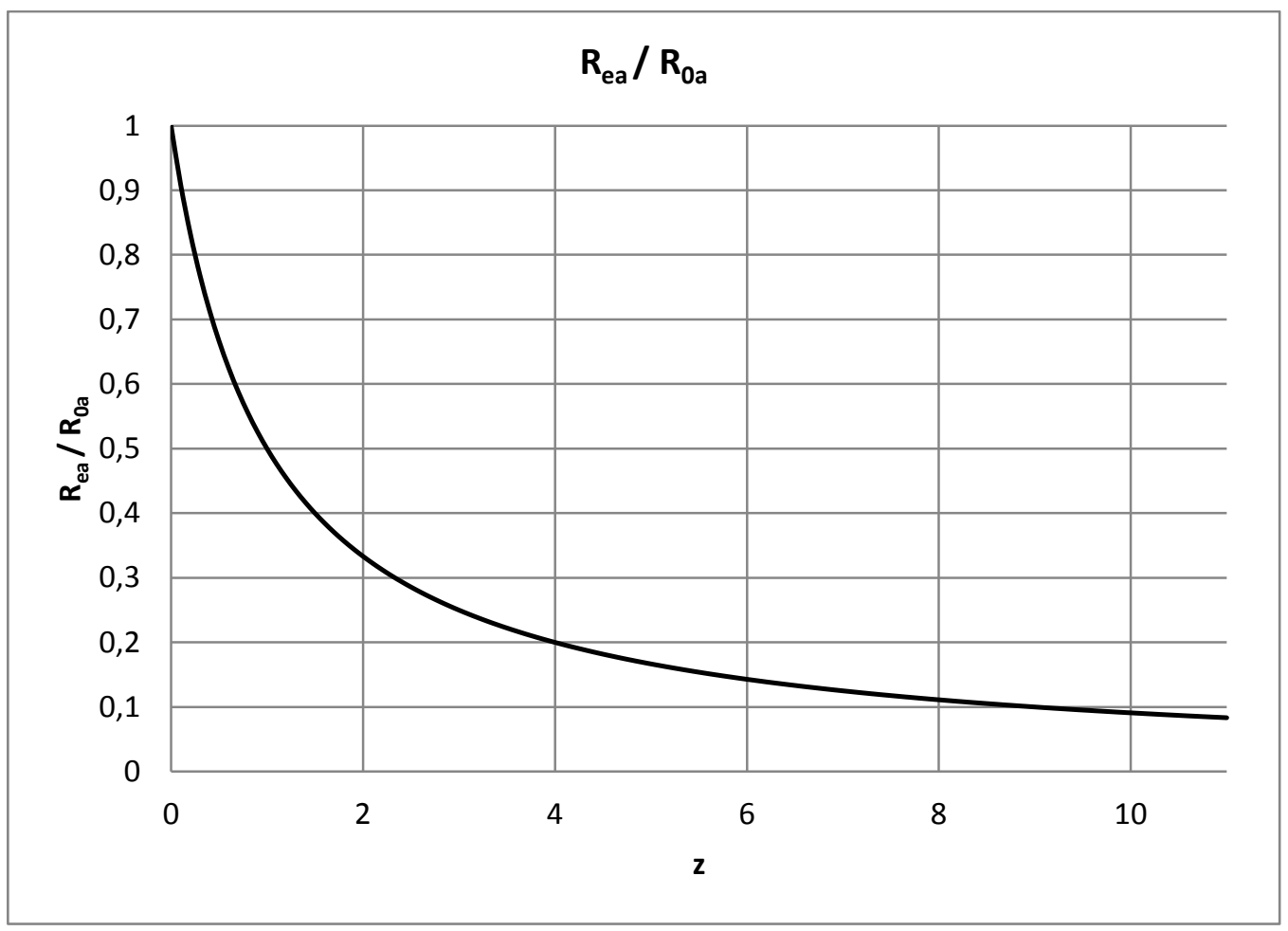

Figure 1. Redshift distance $\mathrm{R}_{\mathrm{ea}}$ normalized to the distance $\mathrm{R}_{0 \mathrm{a}}$.

This distance does not depend on the parameter $\beta_{0}$.

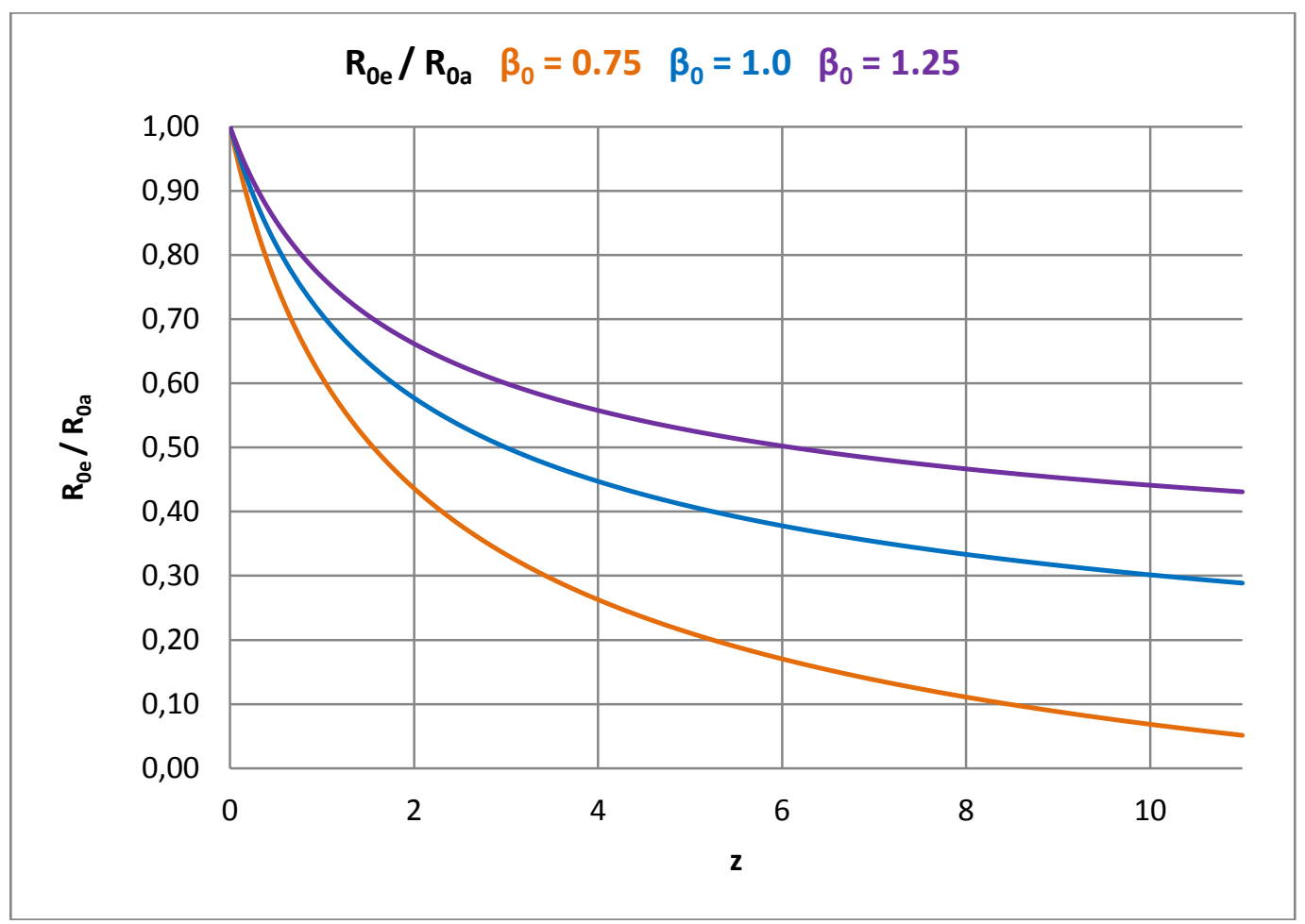

Figure 2. Redshift distance $\mathrm{R}_{0 \mathrm{e}}$ normalized to the distance $\mathrm{R}_{0 \mathrm{a}}$ for various values of the parameter $\beta_{0}$. 


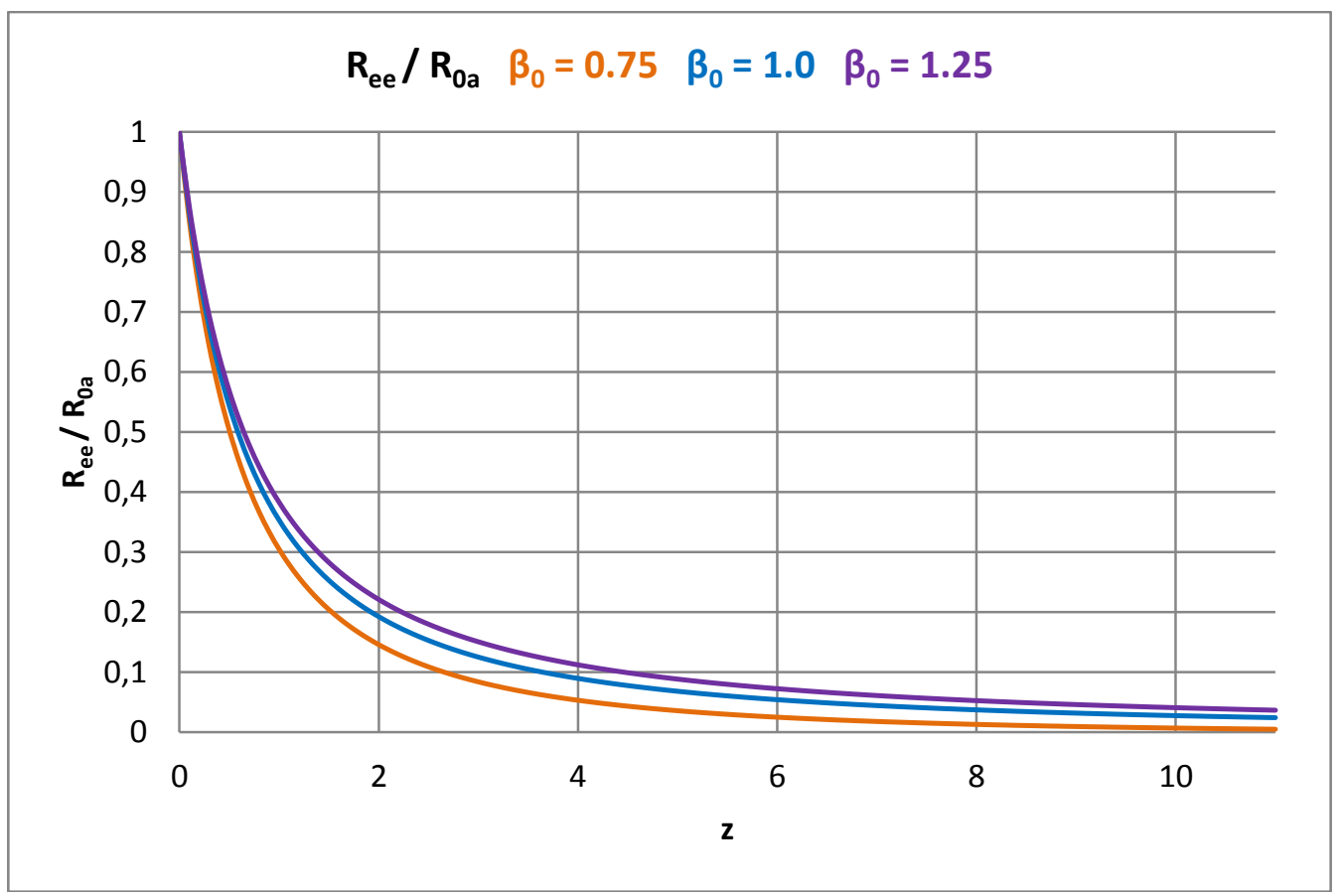

Figure 3. Redshift distance $R_{e e}$ normalized to the distance $R_{0 a}$ for different values of the parameter $\beta_{0}$.

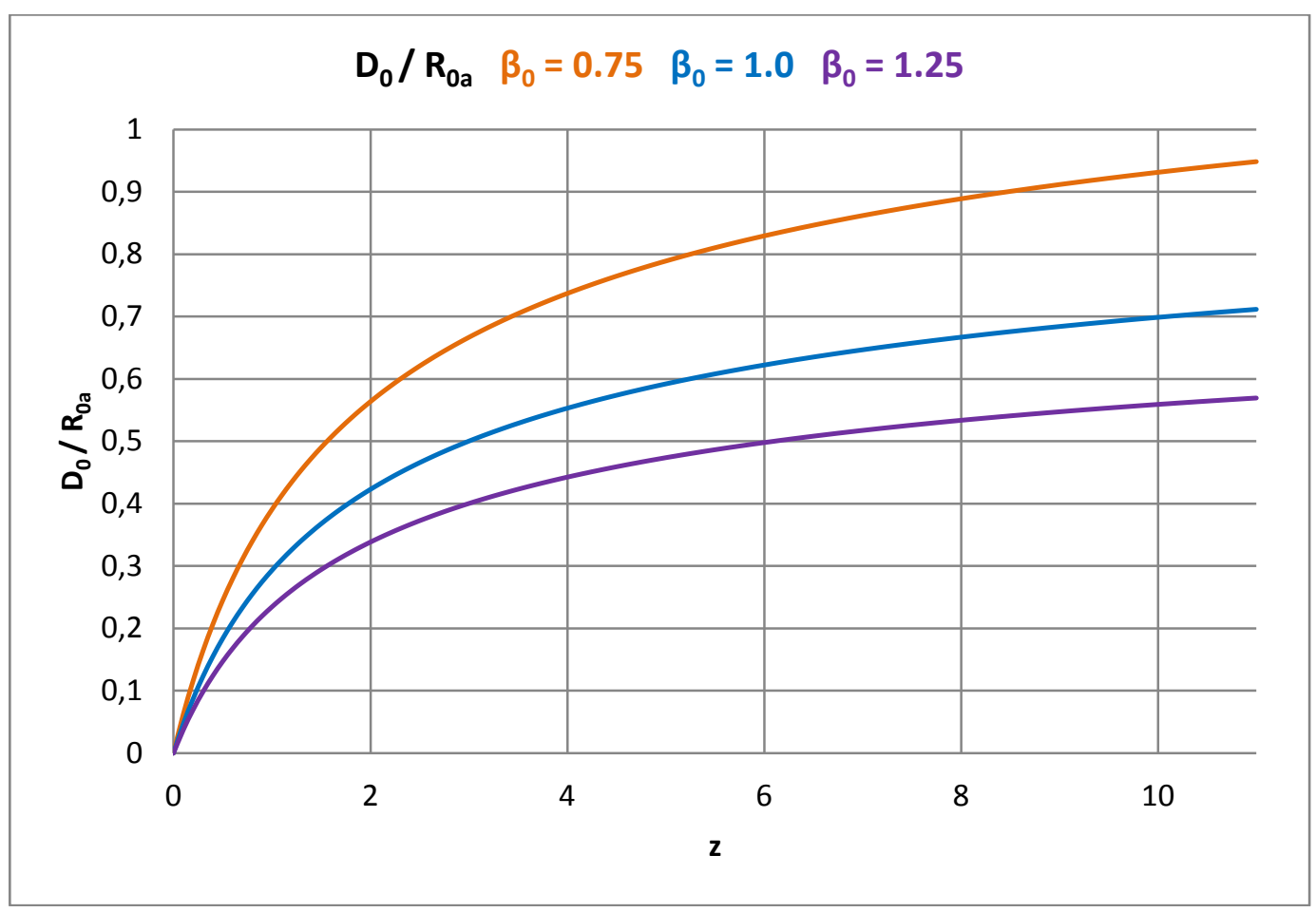

Figure 4. Today's ( $t_{0}$ ) redshift distance $D_{0}$ normalized to the distance $\mathrm{R}_{0 \mathrm{a}}$ for various values of the parameter $\beta_{0}$. 


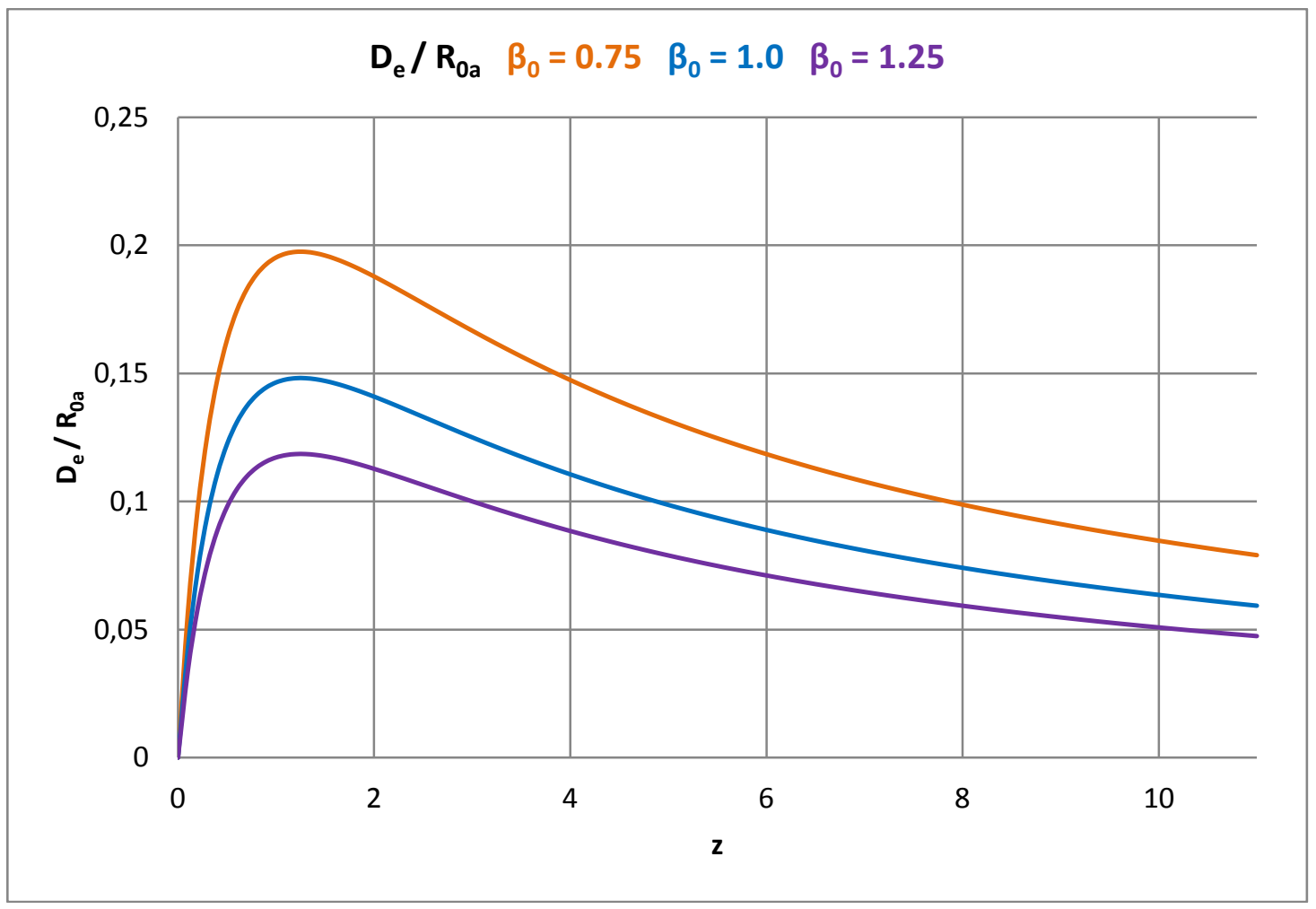

Figure 5. The at that time $\left(t_{e}\right)$ redshift distance $D_{e}$ normalized to the distance $R_{0 a}$ for various values of the parameter $\beta_{0}$.

In the specialist literature, none of these redshift distances are known and they cannot be derived there, respectively.

We will give concrete values for these redshift distances for the galaxy M87 and 27 SNIa below.

\section{Determination of the parameter values}

\subsection{Magnitude-redshift relation}

The apparent magnitude $\mathrm{m}$ depends according to Eq. (I.38) in addition to the measurable redshift $\mathrm{z}$ also on the parameters $\beta_{0}$ and $\mathrm{m}_{0 \mathrm{a}}$.

To find both parameters, the quasar catalog by Véron-Cetty [2] is suitable in which measured redshifts and apparent magnitudes of 132,975 quasars are given.

Fig. 6 shows all these quasars in a single magnitude-redshift diagram, where we have used $\log _{10}(\mathrm{cz})$ as the abscissa. 


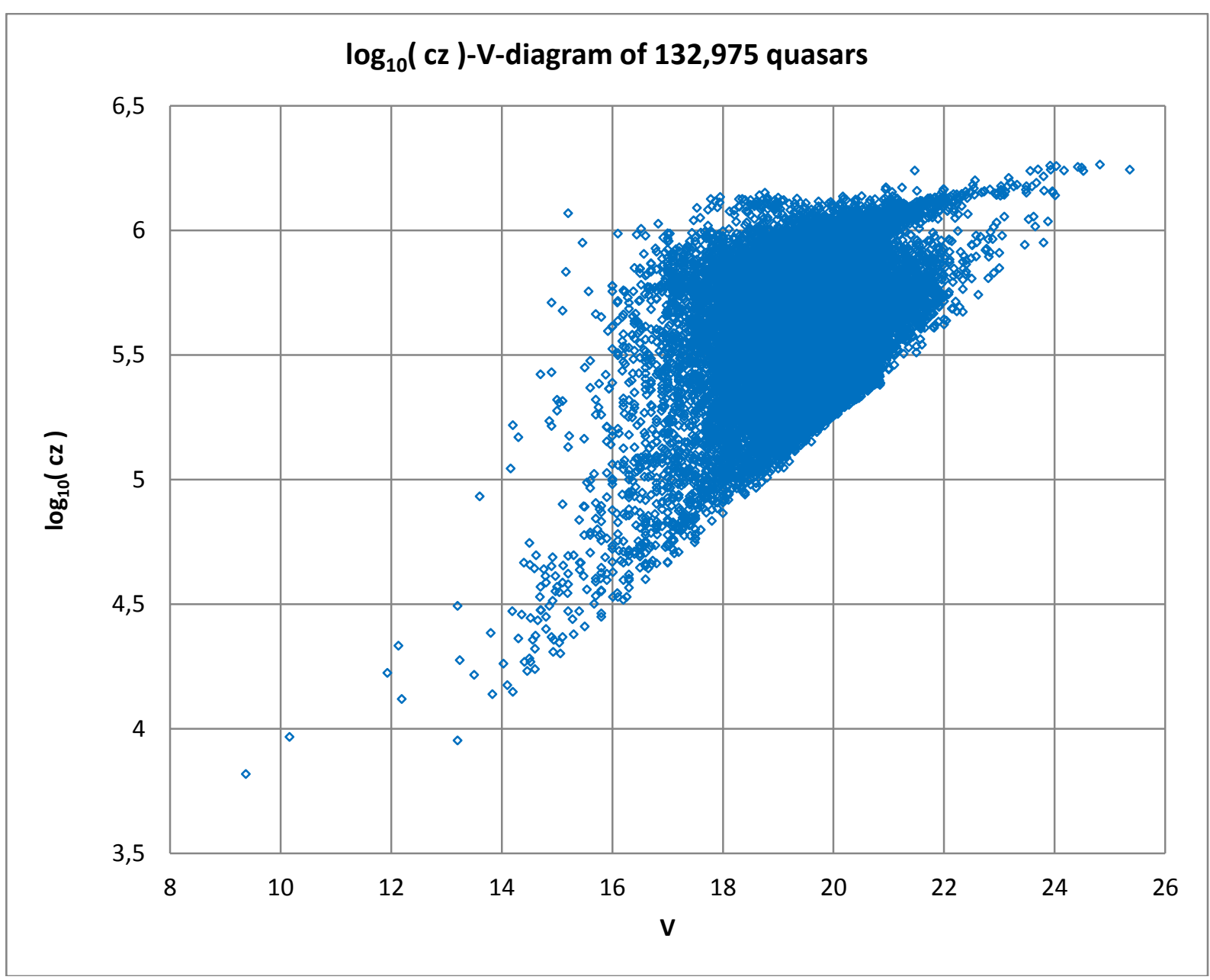

Figure 6. Magnitude-redshift diagram for all 132,975 quasars according to M.-P. Véron-Cetty et al. [2].

A clear edge can be seen on the right side of the accumulation of measurement points, which indicates minimum apparent magnitudes for associated redshifts. The apparent magnitudes are usually up to far to the left of this edge in the diagram.

If we form redshift intervals with mean values of the redshifts and the corresponding mean values for the apparent magnitude, this fact leads to a clear curvature of the mean value curve in the direction of the redshift axis.

The quasars cannot therefore be described in the diagram by a linear curve. This suggests that our redshift distance [i.e. ultimately Eq. (I, 38)] could be suitable for the measured values.

It is precisely this strange magnitude-redshift diagram that has stimulated us to think about cosmological distance determinations for many years [10].

To evaluate the quasar data set, we first create $75 \mathrm{z}$-intervals with 1,773 quasars each. For these intervals we calculate the mean values $\left\langle\mathrm{z}_{\mathrm{i}}\right\rangle$ and the associated mean values $\left\langle\mathrm{m}_{\mathrm{i}}\right\rangle$ of the quasars. For all intervals we also calculate the standard deviations $\sigma_{\mathrm{m}, \mathrm{i}}$ and also the standard deviations $\sigma_{\mathrm{z}, \mathrm{i}}$. The latter, however, do not play a role in the analysis of the data set. The appendix contains the associated table, which also contains all $\sigma_{\mathrm{m}, \mathrm{i}}$.

Figure 7 shows the magnitude-redshift diagram after averaging with all of the standard deviations $\sigma_{\mathrm{m}, \mathrm{i}}$ and $\sigma_{\mathrm{z}, \mathrm{i}}$ calculated by us. 


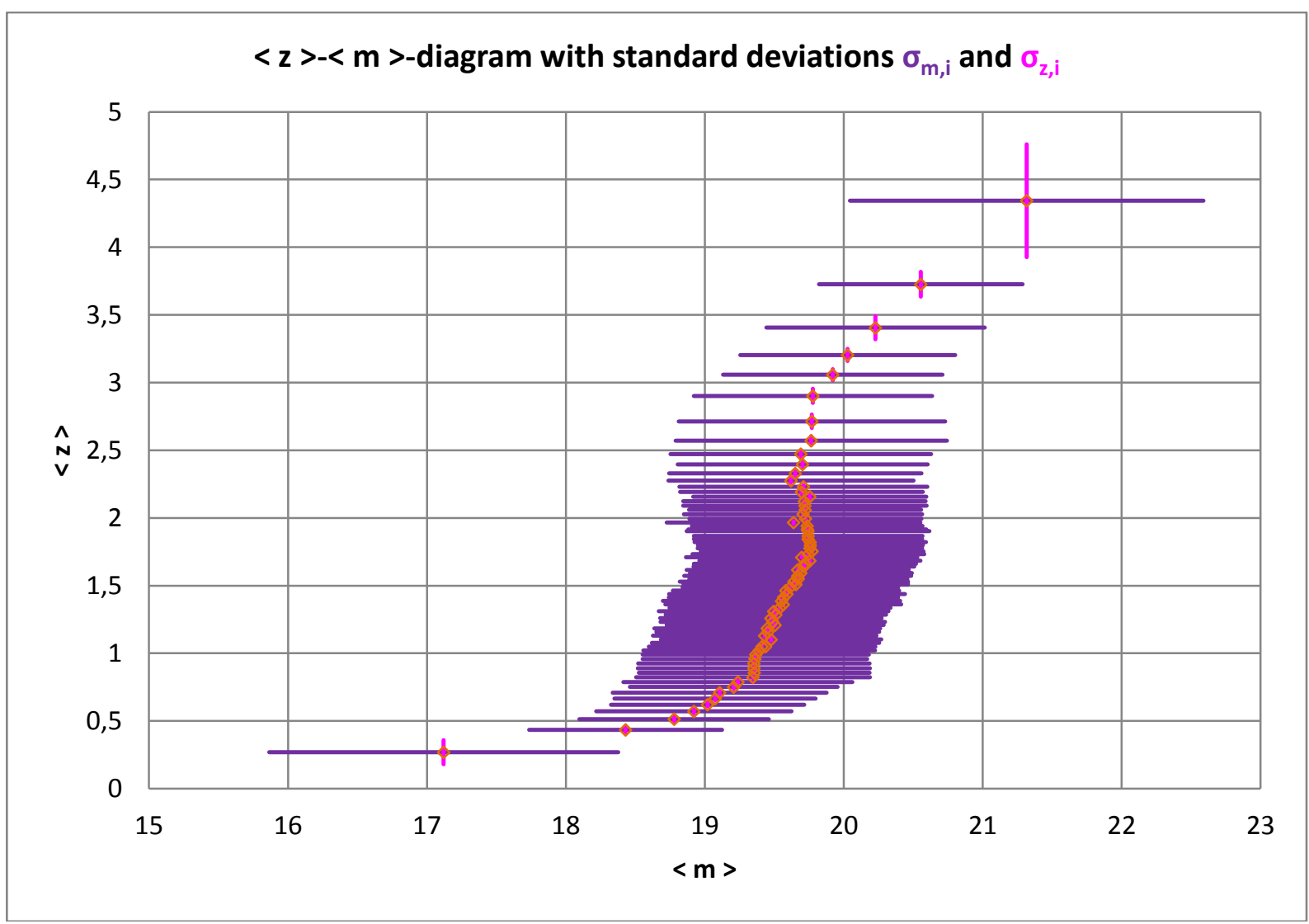

Figure 7. Magnitude-redshift diagram of the mean values $\left\langle\mathrm{z}_{\mathrm{i}}\right\rangle$ and $\left\langle\mathrm{m}_{\mathrm{i}}\right\rangle$ with inserted standard deviations $\sigma_{\mathrm{m}, \mathrm{i}}$ and $\sigma_{\mathrm{z}, \mathrm{i}}$.

The curvature of the curve expected on the basis of Figure 6 can be clearly seen. This curvature should be explained by means of theory. More precisely: The theory has to explain the curvature!

We use the likelihood function

$$
\chi^{2}\left(p_{k}\right)=\sum_{i=1}^{N} \frac{\left[m_{t h, i}\left(p_{k}\right)-m_{o b s, i}\right]^{2}}{\sigma_{m, i}{ }^{2}}
$$

for the evaluation.

$\mathrm{p}_{\mathrm{k}}$ with $\mathrm{k}=1,2$ stands for the two parameters we are looking for, $\beta_{0}$ and $\mathrm{m}_{0 \mathrm{a}}$.

If we use our magnitude-redshift relation (I, 38), the result is concrete

$$
\chi^{2}\left(\beta_{0}, m_{0 a}\right)=\sum_{i=1}^{N} \frac{\left[5 \log _{10}\left[\frac{1}{\beta_{0}}\left(1-\frac{1}{\sqrt{1+z_{i}}}\right)+z_{i}\right]-5 \log _{10}\left(1+z_{i}\right)+m_{0 a}-m_{o b s, i}\right]^{2}}{\sigma_{m, i}^{2}} .
$$

Using the quasar data and using the usual mathematical procedure, we can find the parameters $\beta_{0}=0.7311668$ and $\mathrm{m}_{0 \mathrm{a}}=20.1346$.

Figure 8 shows the result of the mean value formation and the adaptation of the theory to the curvature of the mean value curve. 


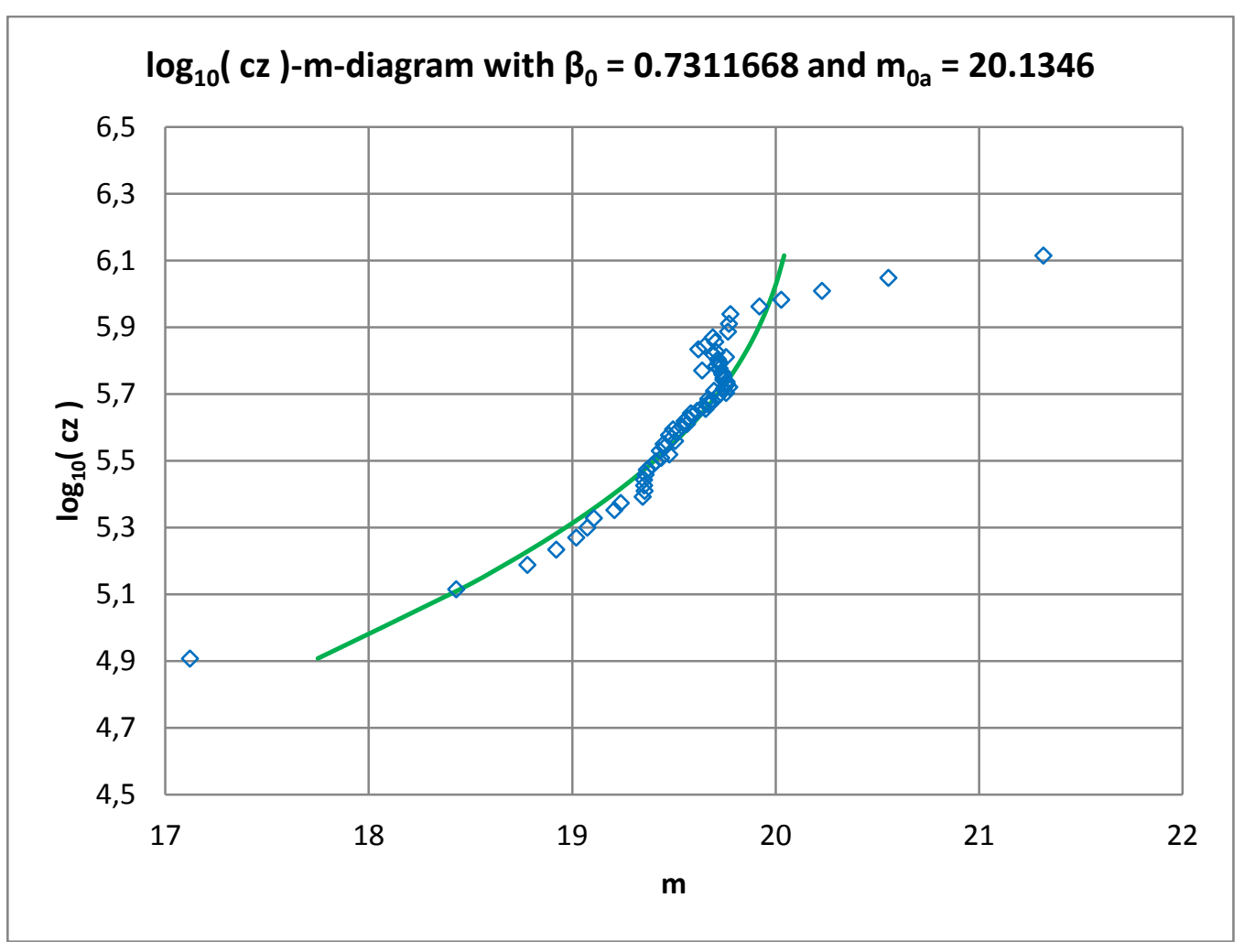

Figure 8. Magnitude-redshift diagram for 132,975 quasars according to M.-P. Véron-Cetty et al. [2].

For comparison:

In Part I of the series of articles we found the somewhat larger value $\mathrm{m}_{0 \mathrm{a}} \approx 20.24$ of today's apparent limit magnitude for much fewer quasars under the justified assumption of $\beta_{0}=1$. There, this value for $\beta_{0}$ best describes the curvature of the measured value curve for large redshifts.

To interpret the measured magnitude-redshift relation:

From our point of view, the quasars came in to being historically slowly as relatively weakly luminous objects at a point in time that corresponds to about $\mathrm{z} \approx 4.3$. The quasars later behaved as the theory expects in flat space and moved with time - i.e. for decreasing redshifts $\mathrm{z}$ - on average along the theoretical curve (in the diagram from top right diagonally to bottom left). The quasars have gradually died out in the recent past and have become relatively bright in the process.

The dependence of the calculated standard deviations $\sigma_{\mathrm{m}, \mathrm{i}}$ on the redshift mean values $\left\langle\mathrm{z}_{\mathrm{i}}\right\rangle$ is shown in Figure 9. 


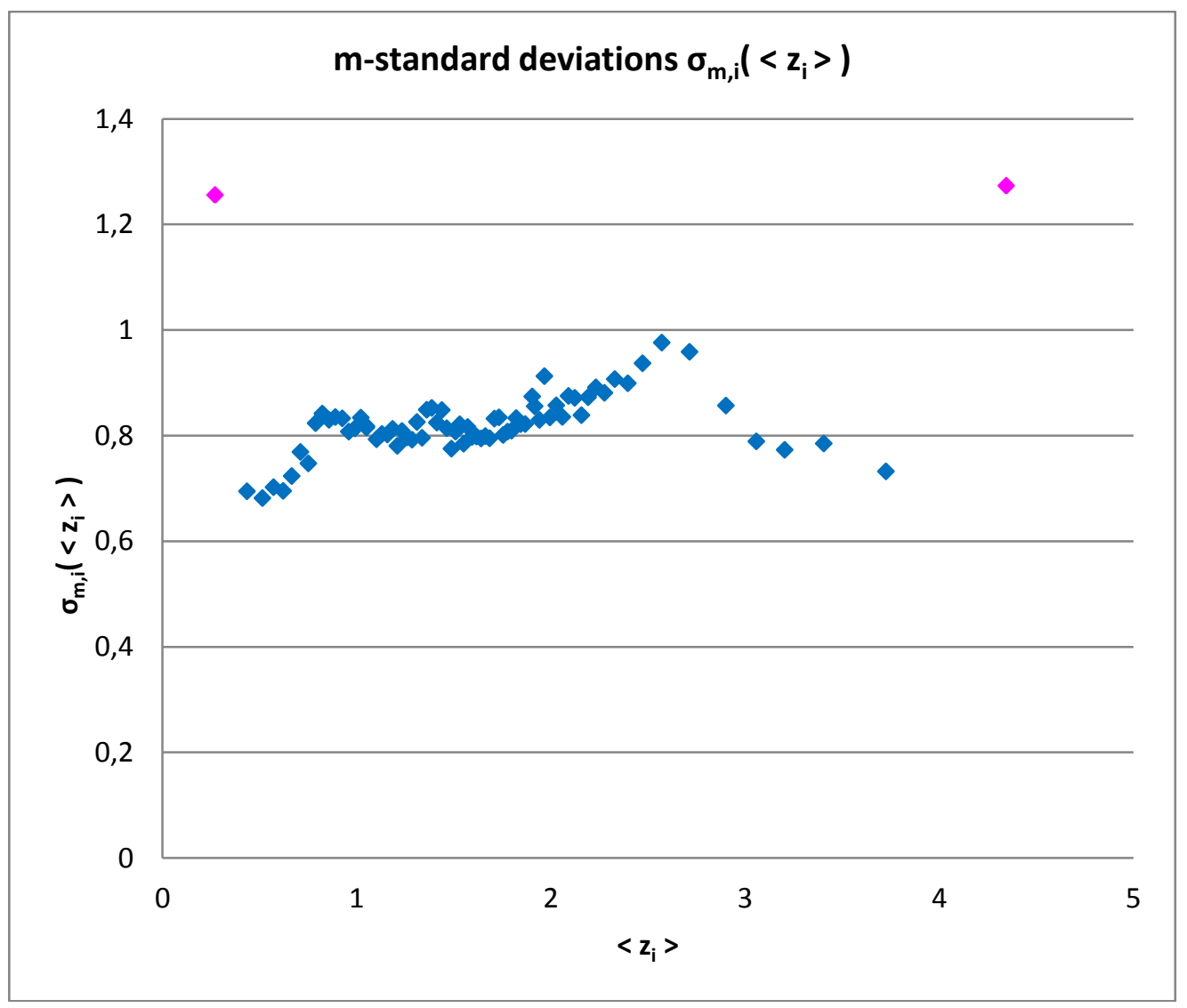

Figure 9. Standard deviations $\sigma_{\mathrm{m}, \mathrm{i}}$ as a function of $\left\langle\mathrm{z}_{\mathrm{i}}\right\rangle$.

If we consider the first and last point in the diagram as outliers and therefore simply do not take them into account when evaluating the magnitude-redshift diagram of the quasars, we find the parameters $\beta_{0}=0.5486497$ and $\mathrm{m}_{0 \mathrm{a}}=19.9555$ for the values.

Because of the differences to the values mentioned above, we might come up with the idea of taking the mean values of each of these. But we will not do that in the following.

\subsection{Number-redshift relation}

We use the following variance to evaluate the number-redshift relation

$$
\chi^{2}\left(p_{k}\right)=\frac{1}{N-1} \sum_{i=1}^{N}\left[N_{t h, i}\left(p_{k}\right)-N_{o b s, i}\right]^{2} .
$$

$\mathrm{p}_{\mathrm{k}}$ with $\mathrm{k}=1,2$ stands for the two parameters we are looking for, $\beta_{0}$ and $\mathrm{N}_{0 \mathrm{a}}$.

If we insert our number-redshift relation $(I, 46)$, the result is concrete

$\chi^{2}\left(\beta_{0}, N_{0 a}\right)=\frac{1}{N-1} \sum_{i=1}^{N}\left\{3 \log _{10}\left[\frac{1}{\beta_{0}}\left(1-\frac{1}{\sqrt{1+z_{i}}}\right)+z\right]-3 \log _{10}\left(1+z_{i}\right)+\log _{10} N_{0 a}-N_{o b s, i}\right\}^{2}$

Using simple mathematics, we find $\mathrm{N}_{0 \mathrm{a}}=146,816$ for the theoretically expected total number of quasars, if we use the value $\beta_{0}=0.7311668$ found via the magnitude-redshift relation.

The expected number is slightly larger than the actual number of quasars measured. This indicates a certain incompleteness of the measurements. 
Figure 10 shows the graphic result.

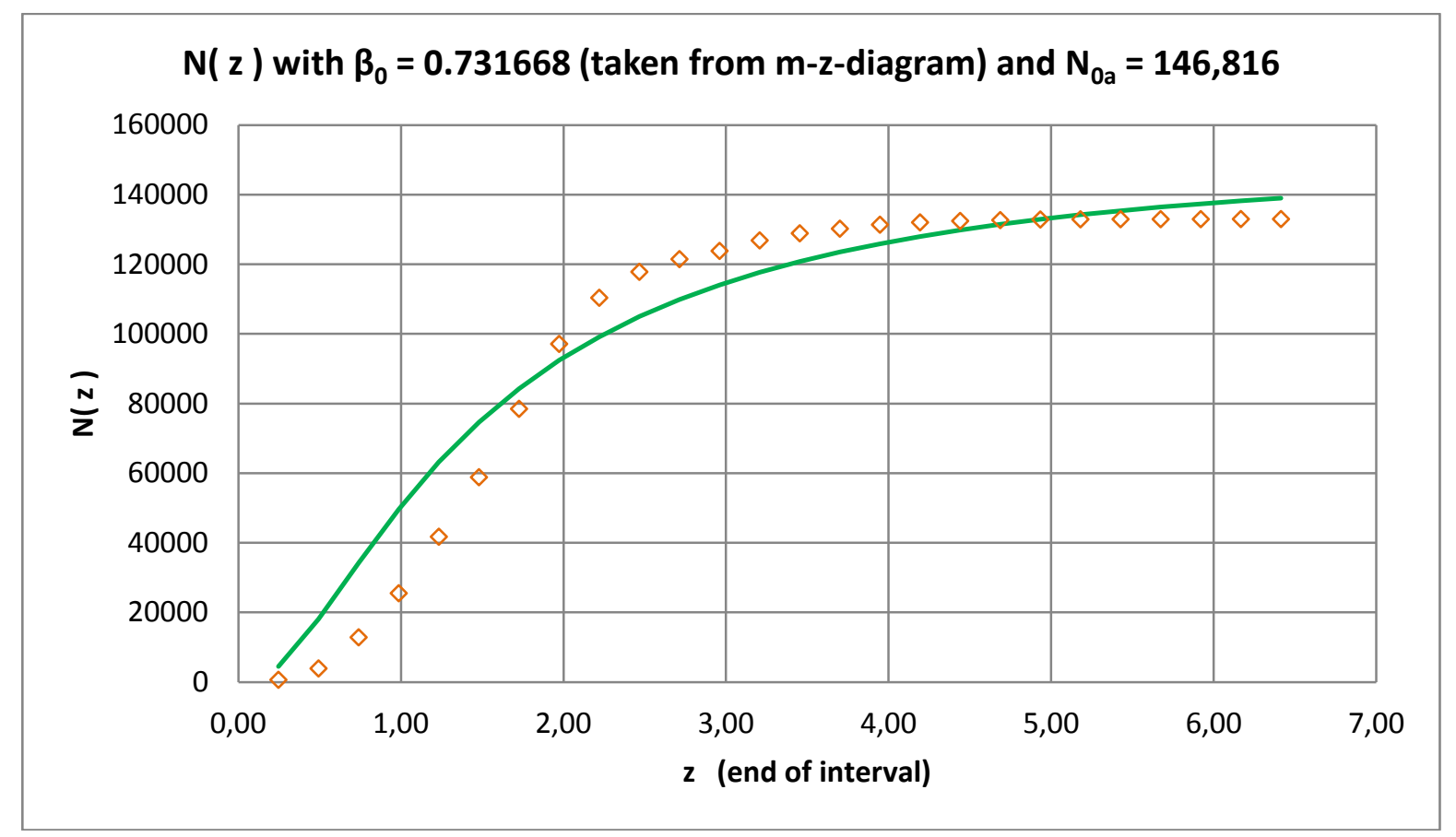

Figure 10. Number-redshift diagram for the 132,975 quasars according to M.-P. Véron-Cetty et al. [2].

Another possibility is to determine both parameters directly via the number-redshift relation, i.e. not to use the value of $\beta_{0}$ from the magnitude-redshift diagram of the quasars. This leads to the parameter values $\mathrm{N}_{0 \mathrm{a}}=159,140$ and $\beta_{0}=0.8653211$. Both values are slightly larger than those noted above.

Overall, we could build a mean value using three different values of $\beta_{0}$. However, we will not make use of this in the following.

\subsection{Angular size-redshift relation}

As in Part I, we use the measurement data from K. Nilsson et al. [3] to find an average linear size of the cosmic objects measured there.

The starting point is the variance

$$
\chi_{\varphi}^{2}\left(p_{k}\right)=\frac{1}{N-1} \sum_{i=1}^{N}\left[\varphi_{t h, i}\left(p_{k}\right)-\varphi_{o b s, i}\right]^{2} .
$$

Here, $p_{k}$ with $k=1,2$ stands for the two parameters we are looking for, $\beta_{0}$ and $\delta / R_{0 a}$.

If we use our angular size-redshift relation (I, 40), the result is concrete

$$
\chi_{\varphi}^{2}\left(\frac{\delta}{R_{0 a}}, \beta_{0}\right)=\frac{1}{N-1} \sum_{i=1}^{N}\left\{\frac{\delta}{R_{0 a}\left[\frac{1}{\beta_{0}}\left(1-\frac{1}{\sqrt{1+z}}\right)+z\right]}-\varphi_{o b s, i}\right\}^{2} .
$$


The comparison of the theory with the measurement data using $\beta_{0}=0.7311668$ results in a value of $\delta / \mathrm{R}_{0 \mathrm{a}}=6.06$ X $10^{-5}$.

Figure 11 shows the graphic result.

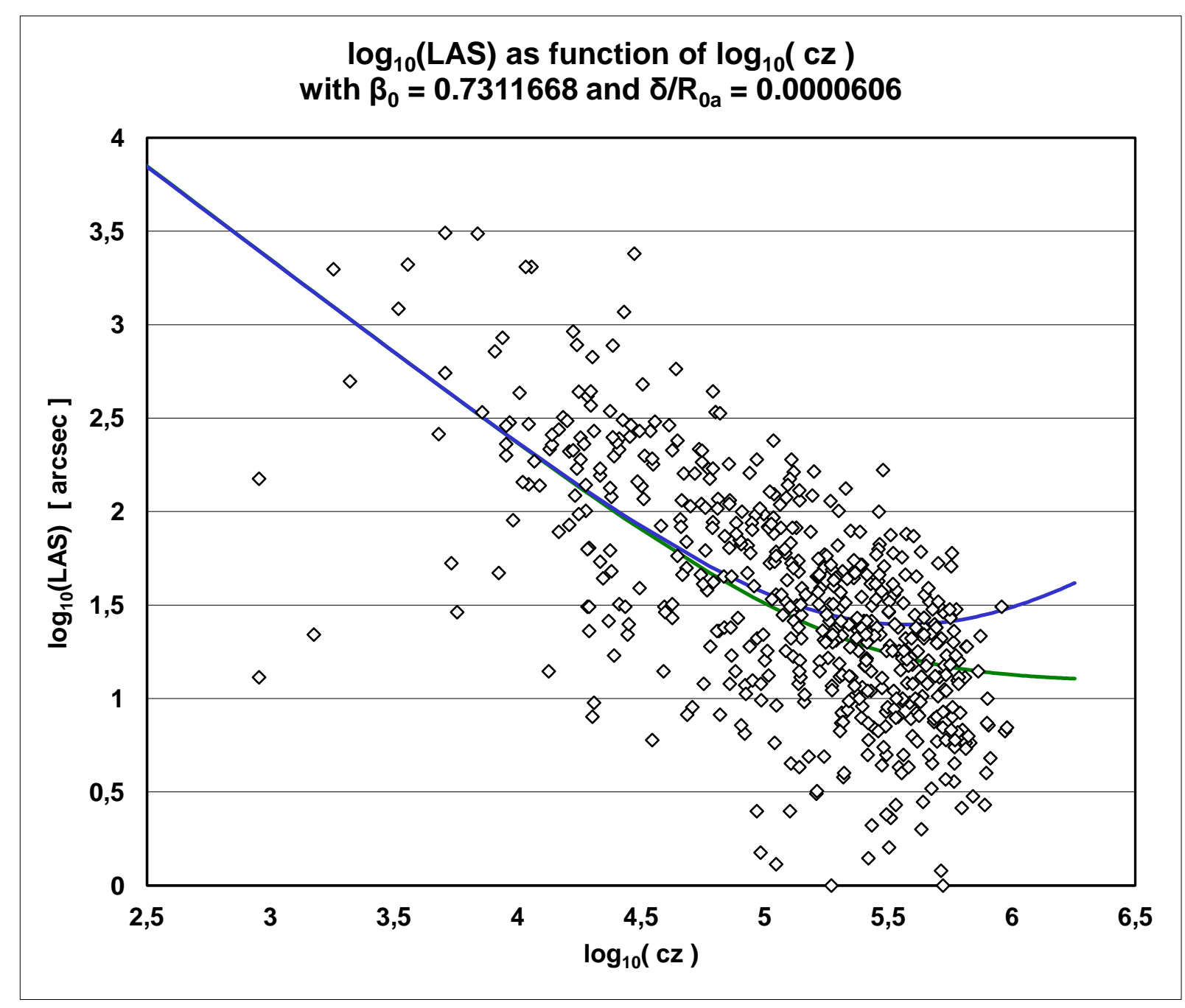

Figure 11. Angular size-redshift diagram according to K. Nilsson et al. [3].

For the purpose of comparison, the theoretical curve from the literature was drawn in (see Part I). This curve cannot explain the position of the measured values in the diagram.

The determination of the linear size $\delta$ requires the knowledge of $\mathrm{R}_{0 \mathrm{a}}$. Because the absolute magnitudes are known for some SNIa (which differ slightly from one another), we can determine $\mathrm{R}_{0 \mathrm{a}}$ using a magnitude-redshift diagram of these objects. We'll do that in the next chapter.

\section{$\underline{3.4 \quad F i x i n g ~ o f ~} \mathbf{R}_{0 a}$ with the help of SNIa}

By W. L. Freedman et al. [4], data from a total of 27 SNIa were made available, with the help of which we can determine both the distance $\mathrm{R}_{0 \mathrm{a}}$ - a current distance - and, as a result, the today's Hubble parameter $\mathrm{H}_{0}$. The data we are interested in are the distance modules $\left(\mu_{\mathrm{TRGB}}\right.$ and $\left.\mu_{\mathrm{Ceph}}\right)$, the maximum apparent magnitudes $\left(\mathrm{m}_{\mathrm{CSP} \_\mathrm{B} 0}\right.$ and $\left.\mathrm{m}_{\mathrm{SC} \_\mathrm{B}}\right)$ and the radial velocities $\mathrm{V}_{\mathrm{NED}}$, from which the redshifts $\mathrm{Z}_{\mathrm{NED}}$ can be calculated.

The methods taken into account in [4] for determining the maximum apparent magnitude and thus the associated absolute magnitude are different, which is why somewhat different values are given for one and the same SNIa. For our purposes, we calculate the mean values from these data and assign them to the relevant SNIa. 
We calculate the absolute magnitudes $\mathrm{M}_{\mathrm{i}}$ of the $\mathrm{SNIa}_{\mathrm{i}}$ using $\left(\mu_{\mathrm{TRGB}}-\mathrm{m}_{\mathrm{CSP} \_\mathrm{B} 0}\right)$ and $\left(\mu_{\mathrm{Ceph}}-\mathrm{m}_{\mathrm{SC} \_\mathrm{B}}\right)$ and then always calculate an average value $\left\langle\mathrm{M}_{\mathrm{i}}\right\rangle$ if both value pairs are specified for one and the same SNIa. From all the absolute magnitudes obtained in this way, we finally form the mean value of the absolute magnitude $\langle\mathrm{M}\rangle \approx-$ 19.245, which enables us to determine the distance $\mathrm{R}_{0 \mathrm{a}}$ with the aid of the parameter $\mathrm{m}_{0 \mathrm{a}}$, which results from the magnitude-redshift diagram of the SNIa. The simple equation for this is

$$
R_{0 a}=10^{\frac{m_{0 a}-M}{5}+1}
$$

The graphic result is shown in Figure 12.

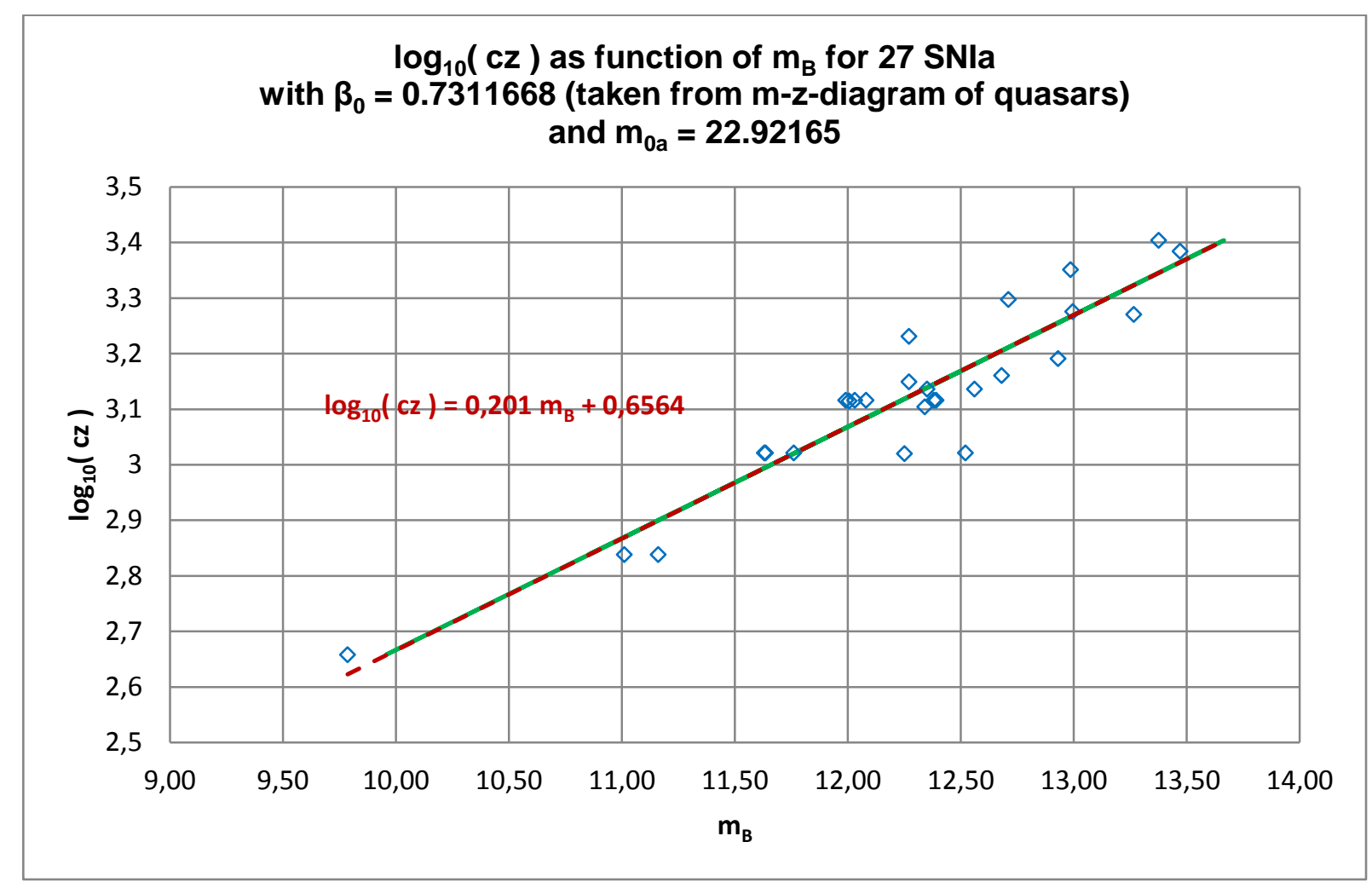

Figure 12. Magnitude-redshift diagram for 27 SNIa according to W. L. Freedman et al. [4].

The theoretical curve lies exactly on the linear trend line (dashed in red), the equation of which is given in the figure.

Using $\mathrm{m}_{0 \mathrm{a}} \approx 22.922$ and the mean value of the absolute brightness $<\mathrm{M}>=-19.245$, the distance $\mathrm{R}_{0 \mathrm{a}} \approx 2,712.48$ $\mathrm{Mpc}$ we are ultimately looking for is the essential result of this data analysis.

With the help of the value of $\mathrm{R}_{0 \mathrm{a}}$ and using the equation (an approximation for small redshifts!)

$$
H_{0 a} \approx \frac{c}{\left(\frac{1}{2 \beta_{0}}+1\right) R_{0 a}}
$$

the today's Hubble parameter $\mathrm{H}_{0} \approx 65.638 \mathrm{~km} /(\mathrm{s} \cdot \mathrm{Mpc})$ results. This value is slightly below the Planck value (2018) with $\mathrm{H}_{0 \text {, Planck }} \approx 67.66 \mathrm{~km} /(\mathrm{s} \cdot \mathrm{Mpc})[5]$.

In Table 8 in the appendix, all the values we used for the magnitude-redshift diagram of the 27 SNIa are compiled.

Starting from the equation 


$$
\frac{1}{\beta_{0}}=\frac{2 c}{R_{0 a} \sqrt{\frac{8 \pi G \rho_{0}}{3}}}
$$

equation (11) results for today's mass density:

$$
\rho_{0}=\frac{3}{2 \pi} \frac{c^{2}}{G} \frac{\beta_{0}{ }^{2}}{R_{0 a}^{2}}
$$

With the theoretical parameters $\beta_{0}$ and $\mathrm{R}_{0 \mathrm{a}}$ determined by us, we find $\rho_{0} \approx 4.843 \times 10^{-27} \mathrm{~g} / \mathrm{cm}^{3}$ for today's matter density in the universe.

Via

$$
M_{F K}=\frac{4 \pi}{3} R_{0 a}{ }^{3} \rho_{0}=\frac{4 \pi}{3} R_{0 a}{ }^{3} \frac{3}{2 \pi} \frac{c^{2}}{G} \frac{\beta_{0}{ }^{2}}{R_{0 a}{ }^{2}} \quad \text { i.e. } \quad M_{F K}=\frac{2 c^{2}}{G} \beta_{0}{ }^{2} R_{0 a}
$$

the constant mass of the Friedmann sphere results in $\mathrm{M}_{\mathrm{FK}} \approx 1.206 \times 10^{56} \mathrm{~g}$.

Because we generally do not consider the accuracy here, we simply specify the decimal places with up to 3 places, whereby the mathematical analysis of the data usually delivers more decimal digits.

With the known value $\mathrm{R}_{0 \mathrm{a}} \approx 2,712.48 \mathrm{Mpc}$ we can calculate the mean linear size of the Nilsson objects [3] to be $\delta \approx 0.164 \mathrm{Mpc}$, because we have found $\delta / \mathrm{R}_{0 \mathrm{a}}=6.06 \times 10^{-5}$ for them.

Using $R_{0 a}$ and $\beta_{0}$, of course, all linear dimensions of these objects can be calculated using their angular size and redshift.

\subsection{Calculation of the further redshift distances for the SNIa and M87}

Because we were able to determine $\mathrm{R}_{0_{a}}$, we can graphically display the further redshift distances in a form that is not normalized to $R_{0 a}$. The result is shown in Figure 13, using the values we found for $\beta_{0}$ and $R_{0 a}$. 


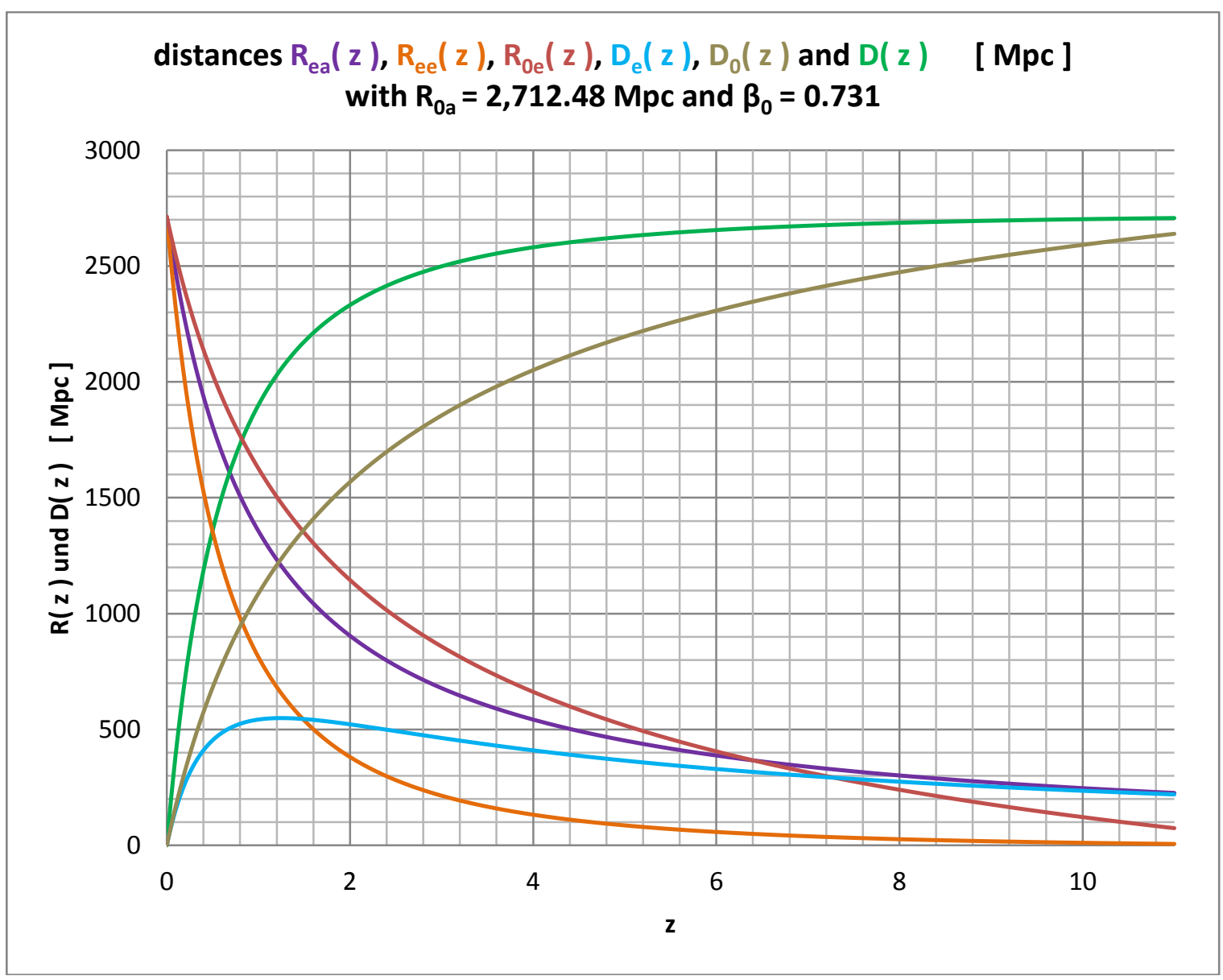

Figure 13. Redshift distance $D$ (light path) and the further redshift distances $D_{i}(i=0, e)$ and $R_{j k}(j=0, e ; k=e$, a) as a function of the redshift up to $\mathrm{z}=11$.

For the representation we have chosen $\mathrm{z} \leq 11$, because currently no cosmic objects with larger measured redshifts are known.

\section{To interpret Figure 13:}

a) For $\mathrm{D} \rightarrow \mathrm{R}_{0 \mathrm{a}}$ the redshift $\mathrm{z}$ goes towards infinity. This means that no observer can observe objects for which is $\mathrm{D} \geq \mathrm{R}_{0 \mathrm{a}} \approx 2,712.48 \mathrm{Mpc}$.

b) As already mentioned in Part $I$, the light path distance $D=R_{0 a}-R_{e e}$ is always greater than the distance differences $\mathrm{D}_{0}$ (today) and $\mathrm{D}_{\mathrm{e}}$ (then).

In particular, the light path $\mathrm{D}$ is not equal to the today's distance $\mathrm{D}_{0}$ between the two astrophysical objects. c) The distances $R_{j k}$ are physical distances from a coordinate origin and develop directly with the change in the scale parameter $\mathrm{a}(\mathrm{t})$ over time. For large redshifts, the scale parameter was correspondingly small and, as a result, the associated distances were also correspondingly small.

d) The distance $D_{e}$ at that time is interesting: It shows a maximum for a specific redshift and only approaches zero for very large redshifts. This is also the reason for the further approximation of $\mathrm{D}_{0}$ to $\mathrm{D}$ only for very large redshifts.

Table 1 summarizes all calculated redshift distances of the 27 SNIa.

\begin{tabular}{|c|c|c|c|c|c|c|c|}
\hline SNIa & $\mathbf{R}_{\text {ea }}$ & $\mathbf{R}_{\text {ee }}$ & $\mathbf{R}_{\mathbf{0 e}}$ & $\mathbf{R}_{\mathbf{0 a}}$ & $\mathbf{D}_{\mathrm{e}}$ & $\mathbf{D}_{\mathbf{0}}$ & $\mathbf{D}$ \\
\hline 1980N & $2,700.72$ & $2,692.70$ & $2,704.43$ & $2,712.48$ & 8.02 & 8.05 & 19.78 \\
\hline 1981B & $2,703.02$ & $2,696.56$ & $2,706.00$ & $2,712.48$ & 6.46 & 6.48 & 15.92 \\
\hline 1981D & $2,700.72$ & $2,692.70$ & $2,704.43$ & $2,712.48$ & 8.02 & 8.05 & 19.78 \\
\hline 1989B & $2,706.26$ & $2,702.02$ & $2,708.23$ & $2,712.48$ & 4.25 & 4.26 & 10.47 \\
\hline 1990N & $2,703.02$ & $2,696.56$ & $2,706.00$ & $2,712.48$ & 6.46 & 6.48 & 15.92 \\
\hline 1994D & $2,703.02$ & $2,696.56$ & $2,706.00$ & $2,712.48$ & 6.46 & 6.48 & 15.92 \\
\hline 1994ae & $2,698.51$ & $2,689.00$ & $2,702.92$ & $2,712.48$ & 9.52 & 9.57 & 23.49 \\
\hline
\end{tabular}




\begin{tabular}{|c|c|c|c|c|c|c|c|}
\hline 1995al & $2,695.53$ & $2,683.98$ & $2,700.87$ & $2,712.48$ & 11.54 & 11.61 & 28.50 \\
\hline $1998 \mathrm{aq}$ & ,700.16 & $2,691.76$ & $2,704.05$ & $2,712.48$ & 8.40 & 8.44 & 70 \\
\hline 1998bu & $2,706.26$ & $2,702.02$ & $2,708.23$ & $2,712.48$ & 4.25 & 4.26 & , \\
\hline 2001el & 2,70 & 2,69 & $2,706.02$ & 2.48 & 6.44 & .46 & . \\
\hline $2002 \mathrm{fk}$ & 2,69 & 2,6 & .00 & 2.48 & 11.41 & 11.48 & 20.17 \\
\hline $2003 \mathrm{du}$ & 2,69 & 2,6 & 2,65 & 48 & 4.78 & 14.90 & 5.01 \\
\hline $2005 \mathrm{cf}$ & 2,6 & 2,6 & 68 & 48 & 3.70 & 13.81 & . \\
\hline 2006dd & 2,70 & 2,6 & .43 & .48 & 8.019 & 8.054 & 19.78 \\
\hline 2007af & 2,6 & 2,6 & 2,7 & .48 & 12.13 & 12.21 & 29.95 \\
\hline 20 & 72 & 2 , & & 48 & 8.02 & 8.05 & 19.78 \\
\hline 200 & 2, & & & 48 & 43 & .49 & 0 \\
\hline 2009ig & 2,68 & 2,6 & 90 & 48 & 15.45 & 15.58 & 38.1 \\
\hline 2011 by & 2,70 & 2,6 & 2,7 & 2.48 & 8.40 & 44 & 20.7 \\
\hline $2011 \mathrm{fe}$ & 2,70 & 2,7 & .67 & 2.48 & 2.81 & 2.81 & 6.9 \\
\hline $2011 \mathrm{iv}$ & 2,70 & 2,6 & 2,70 & 2.48 & 8.02 & 8.05 & 19.7 \\
\hline $2012 \mathrm{cg}$ & $2,703.02$ & $2,696.56$ & $2,706.00$ & 2.48 & 6.46 & 6.48 & 15.9 \\
\hline $2012 \mathrm{fr}$ & $2,700.75$ & $2,692.76$ & $2,704.45$ & $2,712.48$ & 7.99 & 8.03 & 19.7 \\
\hline $2012 \mathrm{ht}$ & $2,699.45$ & $2,690.58$ & $2,703.56$ & $2,712.48$ & 8.88 & 8.92 & 21.9 \\
\hline 2013dy & $2,699.79$ & $2,691.13$ & 2,70 & $2,712.48$ & 8.65 & 8.69 & 21.3 \\
\hline $2015 \mathrm{~F}$ & $2,701.03$ & $2,693.23$ & $2,704.64$ & $2,712.48$ & 7.81 & 7.84 & 19. \\
\hline
\end{tabular}

Table 1. Redshift distance $D$ and the further redshift distances $D_{i}$ and $R_{j k}$ of all 27 SNIa.

\section{To interpret the distances from Table 1:}

For a more detailed explanation, we take the SNIa 2006dd, for example, and use it to interpret the meaning of the distances in the table.

The "light-travel time" always means the time interval between the emission of light (time $t_{e, 2006 d d}$ ) by the SNIa 2006dd and today $\left(\mathrm{t}_{0}\right)$, i.e. $\Delta \mathrm{t}=\mathrm{t}_{0}-\mathrm{t}_{\mathrm{e}}$,2006dd. This light-travel time is generally different for all observable cosmic objects, here especially for the individual SNIa we have considered.

a) Today's ( $\mathrm{t}_{0}$ ) distance between the selected SNIa and us as observers is $\mathrm{D}_{0} \approx 8.054 \mathrm{Mpc}$.

b) The then $\left(\mathrm{t}_{\mathrm{e}}\right)$ distance between this SNIa and us as observers was $\mathrm{D}_{\mathrm{e}} \approx 8.019 \mathrm{Mpc}$.

According to this, the distance between the two cosmic objects has increased by about $0.035 \mathrm{Mpc}$ during the light-travel time $\Delta \mathrm{t}=\mathrm{t}_{0}-\mathrm{t}_{\mathrm{e}, 2006 \mathrm{dd}}$.

c) The SNIa has been shifted expansively away from the origin of the coordinates by $\Delta \mathrm{R}_{\mathrm{e}}=\mathrm{R}_{0 \mathrm{e}}-\mathrm{R}_{\mathrm{ee}} \approx 11.73$ Mpc during the light-travel time due to the time-dependent scale parameter a(t).

d) The galaxy with us as observers has been expansively shifted away from the origin of the coordinates by $\Delta \mathrm{R}_{\mathrm{a}}$ $=\mathrm{R}_{0 \mathrm{a}}-\mathrm{R}_{\mathrm{ea}} \approx 17.765 \mathrm{Mpc}$ during the $\mathrm{t}$ light-travel time travel due to $\mathrm{a}(\mathrm{t})$.

The difference between the two displacement distances is of course the increase in the distance between the two cosmic objects noted above.

e) The light path covered by the photons within the time $\Delta \mathrm{t}=\mathrm{t}_{0}-\mathrm{t}_{\mathrm{e}, \text { 2006dd }}$ (redshift distance) is $\mathrm{D} \approx 19.78 \mathrm{Mpc}$. It is unequal to the other mentioned distances $\mathrm{D}_{\mathrm{i}}$ and also greater than these.

\subsection{Evaluation of the data from the black hole in M87}

For the sake of simplicity, we summarize the data from the literature on the galaxy M87 with the black hole (BH) in it in the first line of Table 2 \{s. [6] and [7]\}.

The second line lists the data specified here, which usually differ from those in the literature.

\begin{tabular}{|c|c|c|c|c|c|c|c|}
\hline & $\mathbf{D}[\mathbf{M p c}]$ & $\mathbf{M}_{\mathbf{B}}$ [ mag ] & $\mathbf{z}$ & $\mathbf{m}_{\mathbf{B}}[\mathbf{m a g}]$ & $\boldsymbol{\Theta}_{\mathbf{B H}}[\boldsymbol{\mu a s}]$ & $\boldsymbol{\delta} / \mathbf{2}=\mathbf{R}_{\mathbf{S}}[\mathbf{p c}$ ] & $\mathbf{M}_{\mathbf{B H}}[\mathbf{g}$ ] \\
\hline literature & $16.9 / 16.8$ & -23.5 & 0.004283 & 9.6 & 42 & & $1.2928 \mathrm{E}+43$ \\
\hline we & 19.45 & -21.84 & & & & 0.227 & $2.3584 \mathrm{E}+45$ \\
\hline
\end{tabular}

Table 2. Summary of data from galaxy M87 with the black hole in it. 
The theory was adapted to the measured angle size $\Theta_{\mathrm{BH}}$ from the literature. Overall, a larger redshift distance D, a smaller absolute magnitude $\mathrm{M}_{\mathrm{B}}$ and a significantly larger mass $\mathrm{M}_{\mathrm{BH}}$ of the black hole follow.

Table 3 lists the values found by means of our theory for all redshift distances $R_{j k}, D_{i}$ and for $D$.

\begin{tabular}{|c|c|c|c|c|c|c|c|}
\hline$[\mathrm{Mpc}]$ & $\mathbf{R}_{\mathbf{e a}}$ & $\mathbf{R}_{\mathrm{ee}}$ & $\mathbf{R}_{\mathbf{0 e}}$ & $\mathbf{R}_{\mathbf{0 a}}$ & $\mathbf{D}_{\mathrm{e}}$ & $\mathbf{D}_{0}$ & $\mathbf{D}$ \\
\hline $\mathbf{w e}$ & $2,700.92$ & $2,693.03$ & $2,704.56$ & $2,712.48$ & 7.89 & 7.92 & 19.45 \\
\hline literature & $\mathbf{- - -}$ & --- & --- & --- & $\mathbf{- - -}$ & $\mathbf{- - -}$ & 16.8 \\
\hline
\end{tabular}

Table 3. Redshift distances $D_{i}, D$ and $R_{j k}$ from the black hole in $M 87$.

From these values, the expansion-related shifts in distance of the galaxy M87 and of the galaxy with us as observers can be calculated, which took place during the time of light travel.

The theory from the literature does not know the first 5 listed distances. It can therefore not be calculated using this theory and also not determine in terms of value.

The distance D differs because of the physical meaning: In our theory, D is the real physical light path, which is not the case in the literature.

We briefly interpret the meaning of the distances in Table 3, whereby the light-travel time is again defined as above:

a) Today's $\left(\mathrm{t}_{0}\right)$ distance between the black hole $(\mathrm{BH})$ or the galaxy M87 and us as observers is $\mathrm{D}_{0} \approx 7.92 \mathrm{Mpc}$.

b) The then ( $\mathrm{t}_{\mathrm{e}}$ ) distance between the $\mathrm{BH}$ (or M87) and us as observers was $\mathrm{D}_{\mathrm{e}} \approx 7.89 \mathrm{Mpc}$.

Accordingly, the distance between the two cosmic objects has increased by about $0.03 \mathrm{Mpc}$ during the lighttravel time $\Delta \mathrm{t}=\mathrm{t}_{0-} \mathrm{t}_{\mathrm{e}}, \mathrm{BH}, \mathrm{M} 87$.

c) The BH (or M87) has been shifted expansively away from the origin of the coordinates by $\Delta \mathrm{R}_{\mathrm{e}}=\mathrm{R}_{0 \mathrm{e}}-\mathrm{R}_{\mathrm{ee}} \approx$ $11.53 \mathrm{Mpc}$ during the light-travel time due to the time-dependent scale parameter a(t).

d) The galaxy with us as observer was expansively shifted away from the origin of the coordinates by $\Delta \mathrm{R}_{\mathrm{a}}=\mathrm{R}_{0 \mathrm{a}}$ - $\mathrm{R}_{\mathrm{ea}} \approx 11.57 \mathrm{Mpc}$ during the light-travel time due to $\mathrm{a}(\mathrm{t})$.

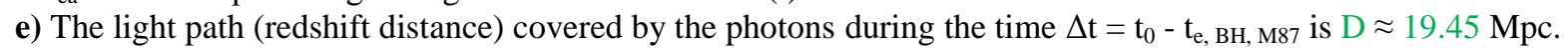
It is unequal to the other mentioned distances $\mathrm{D}_{\mathrm{i}}$ and also greater than these.

Figure 14 shows the various calculated distances in a clear form.

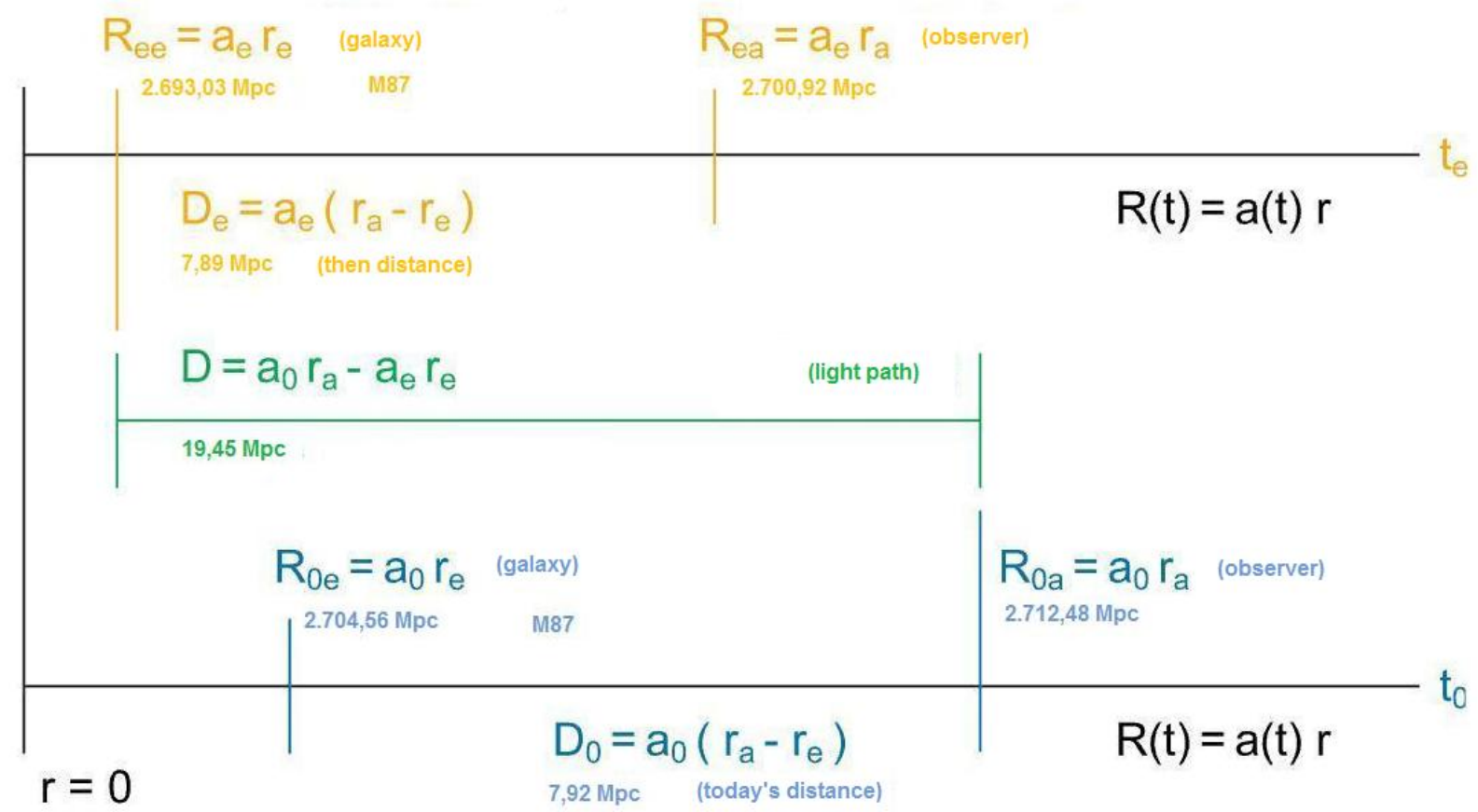


Figure 14. Visualization of the distances $D_{i}, D$ and $R_{j k}$ with regard to $M 87$ and observer.

The distances are not drawn to scale here.

\subsection{Maximum values known today: Galaxy UDFj-39546284 and Quasar J0313}

The galaxy UDFj-39546284 [8] currently holds the record among the galaxies with a redshift of $\mathrm{z}=10.3$, while the quasar J0313 [9] with $\mathrm{z}=7.642$ holds the analog record among the quasars.

Table 4 shows the corresponding distances $\mathrm{R}_{\mathrm{jk}}$, $\mathrm{D}_{\mathrm{i}}$ and $\mathrm{D}$ together.

\begin{tabular}{|c|c|c|c|c|c|c|c|c|c|}
\hline object name & $\mathbf{Z}$ & $\mathbf{D}$ & $\mathrm{D}_{0}$ & $\mathrm{D}_{\mathrm{e}}$ & $\mathbf{R}_{\mathrm{ee}}$ & $\mathbf{R}_{\text {0e }}$ & $\mathbf{R}_{\text {ea }}$ & $\mathbf{R}_{\text {0a }}$ & object \\
\hline J0313 & 7.642 & $2,681.858$ & $1,789.782$ & 207.103 & 30.622 & 264.636 & 313.872 & $2,712.480$ & quasar \\
\hline UDFj-39546284 & 10.300 & $2,703.075$ & $1,905.566$ & 168.634 & 9.405 & 106.281 & 240.042 & $2,712.480$ & galaxy \\
\hline
\end{tabular}

Table 4. All calculated redshift distances $\mathrm{R}_{\mathrm{jk}}, \mathrm{D}_{\mathrm{i}}$ and $\mathrm{D}$ for the two cosmic objects with the maximum redshifts.

Table 5 summarizes the spatial shifts of the objects with respect to the coordinate origin due to the expansion during the associated light travel times.

\begin{tabular}{|c|c|c|c|}
\hline object name & $\mathbf{R}_{\text {0e }}-\mathbf{R}_{\text {ee }}$ & $\mathbf{R}_{\text {0a }}-\mathbf{R}_{\text {ea }}$ & object \\
\hline J0313 & 234.014 & $2,398.608$ & quasar \\
\hline UDFj-39546284 & 96.876 & $2,472.438$ & galaxy \\
\hline
\end{tabular}

Table 5. Expansion-related shifts in the distance of the quasar and the galaxy.

We have already explained above how the tables are to be interpreted.

Figure 15 shows the distances $D_{i}$ and $D$ of the 3 special astrophysical objects analyzed here in a diagram, whereby we have entered all numerical values for the distances in Mpc. 


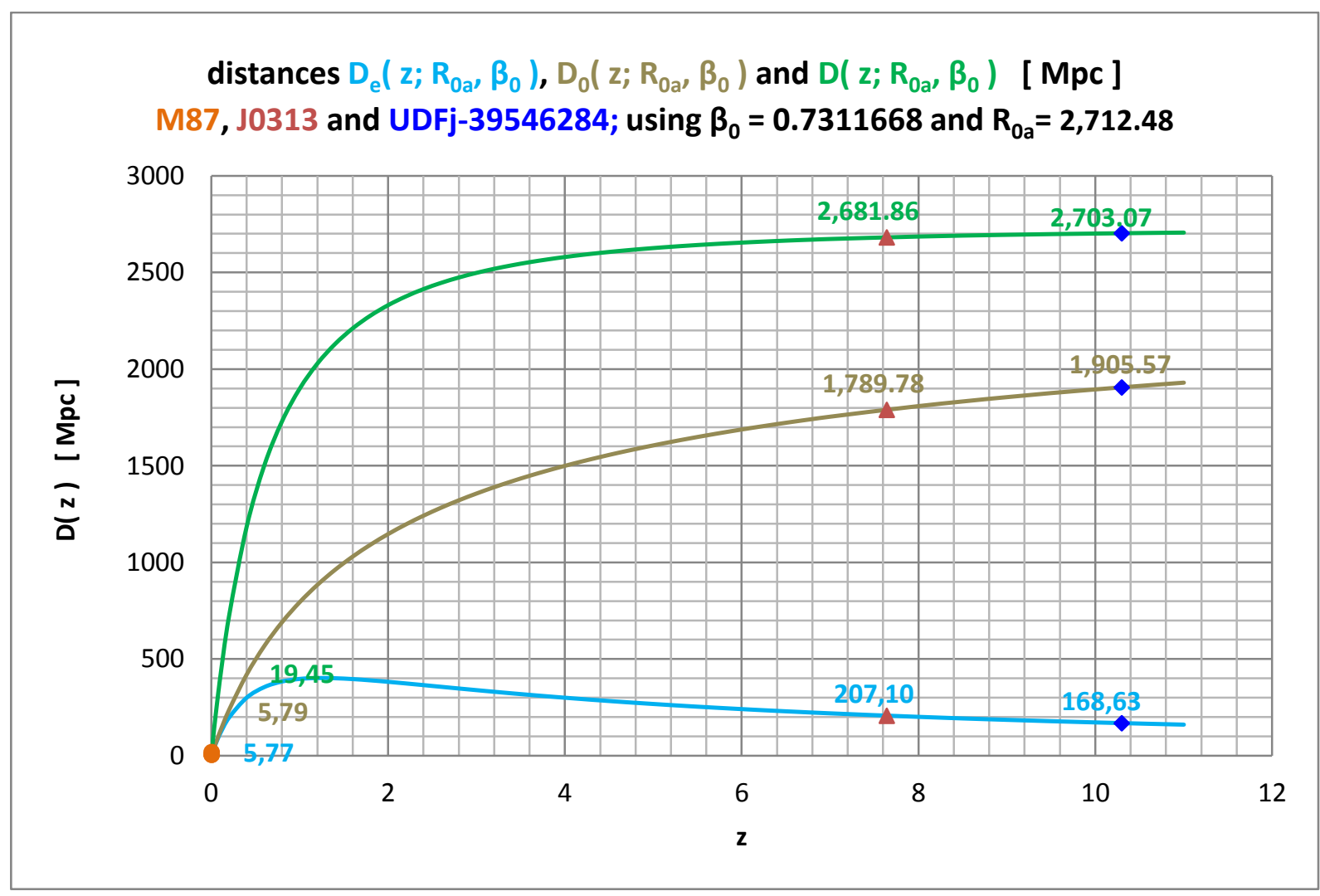

Figure 15. All distances $D_{i}$ and D for M87, J0313 and UDFj-39546284.

The middle curve shows the current distances $\mathrm{D}_{0}$ of the objects from us as observers. These distances are clearly smaller than the associated light paths D.

\section{Final considerations}

\subsection{Hubble parameter}

At this point we explicitly point out that our equation of today's Hubble parameter - which also only applies to very small redshifts - differs significantly from the definition (!) used in the literature:

$$
H_{0 a} \approx \frac{1}{\left(\frac{1}{2 \beta_{0}}+1\right)} \frac{c}{R_{0 a}} \quad(w e)
$$

and

$$
H_{0, \text { lit }}=\frac{\dot{a}_{0}}{a_{0}}=\frac{\dot{a}_{0} r_{a}}{a_{0} r_{a}}=\frac{\dot{R}_{0 a}}{R_{0 a}} \quad r_{a}=\text { const } \quad \text { (literature). }
$$

For an arbitrary point in time $\mathrm{t}$ this is 
$H_{a}(t) \approx \frac{1}{\left(\frac{1}{2 \beta(t)}+1\right)} \frac{c}{a(t) r_{a}}=\frac{1}{\left[\sqrt{\frac{a(t) r_{a}}{R_{S}}}+1\right]} \frac{c}{a(t) r_{a}}$

because of $\frac{1}{\beta(t)}=2 \sqrt{\frac{a(t) r_{a}}{R_{S}}}$ with $\frac{c}{r_{a}}=$ const $\frac{r_{a}}{R_{S}}=$ const $\quad R_{S}=$ const

and

$H_{a, l i t}(t)=\frac{\dot{a}(t)}{a(t)} \frac{r_{a}}{r_{a}} \quad($ literatur $)$.

The index a generally indicates the proximity to the observer $\left(r=r_{a}\right)$.

In our theory, the numerator contains the constant physical speed of light $\mathrm{c}$ in a vacuum, while the current, i.e. variable, spatial expansion speed (da/dt) can be found at this point in the literature.

In the more recent past - time $t_{x}$ - our distance from the coordinate origin $R_{x a}<R_{0 a}$ was slightly smaller than the current one and the Hubble parameter was therefore correspondingly larger (also via the parameter $\beta_{\mathrm{x}}$ ).

In the case of the Hubble parameter in literature, the - actually non-physical - spatial expansion speed da/dt can have been arbitrarily large and, in addition, the scale parameter a(t) arbitrarily small.

Both types of Hubble parameters therefore show completely different behavior!

In addition, our Hubble parameter is actually made up of physical quantities, while the Hubble parameter in the literature is only defined using the non-physical scale parameter a $(t)$, even if the latter can be assigned a suitable unit of measurement - e.g. Mpc. This means that a $(t)$ per se is not a physical distance. This meaning only applies to the real physical distance $R(t)=a(t) r$ and the differences that can be calculated from it.

The Hubble parameter is the proportionality factor between the Hubble speed V $=\mathrm{cz}$ and a distance, i.e. the actual Hubble law applies

$$
V=c z=H_{0 a} D \approx \frac{1}{\left(\frac{1}{2 \beta_{0}}+1\right)} \frac{c}{R_{0 a}} D
$$

and

$$
V_{l i t}=c z=H_{0, l i t} D_{l i t}=\frac{\dot{a}_{0}}{a_{0}} D_{l i t}=\frac{\dot{R}_{0 a}}{R_{0 a}} D_{l i t} \quad \text { (literature) } .
$$

For the redshift $\mathrm{z}$ it simply follows

$$
z=\frac{H_{0 a}}{c} D \approx \frac{1}{\left(\frac{1}{2 \beta_{0}}+1\right)} \frac{D}{R_{0 a}} \quad(\text { we })
$$

and

$$
z=\frac{H_{0, l i t}}{c} D_{l i t}=\frac{\dot{a}_{0}}{c} \frac{D_{l i t}}{a_{0}}=\frac{\dot{R}_{0 a}}{c} \frac{D_{l i t}}{R_{0 a}} \quad \text { (literature) } .
$$

In the literature, the redshift $\mathrm{z}$ is therefore dependent on the ratio of the current speed of the observer (his galaxy) related to the origin of the coordinates to the speed of light in the product with the ratio of an object distance $\mathrm{D}_{\text {lit }}$ and the current distance of the observer's galaxy from the origin of the coordinates. 
Our redshift, on the other hand, is dependent on the ratio of the light path distance D and the current distance of the observer galaxy from the coordinate origin $\mathrm{R}_{0 \mathrm{a}}$ and is besides proportional to the factor that contains the parameter $\beta_{0}$.

Using the parameter $\beta_{0}$

$$
\frac{1}{\beta_{0}}=2 \sqrt{\frac{R_{0 a}}{R_{S}}} \quad \text { with } \quad R_{S}=\frac{2 M G}{c^{2}}
$$

we see in our case

$$
z=\frac{H_{0 a}}{c} D \approx \frac{1}{\left(\sqrt{\frac{R_{0 a}}{R_{S}}+1}\right)} \frac{D}{R_{0 a}} \quad \text { (we) }
$$

i.e. an direct dependence on the $S$ chwarzschild radius $R_{S}$, or more precisely on the ratio $R_{0 a}$ to $R_{S}$.

Overall, it is somewhat unclear in the literature what exactly corresponds to the distance $\mathrm{D}_{\text {lit }}$.

Figure 16 shows the difference between the non-approximated redshift distance D and the linear Hubble redshift distance that is approximated.

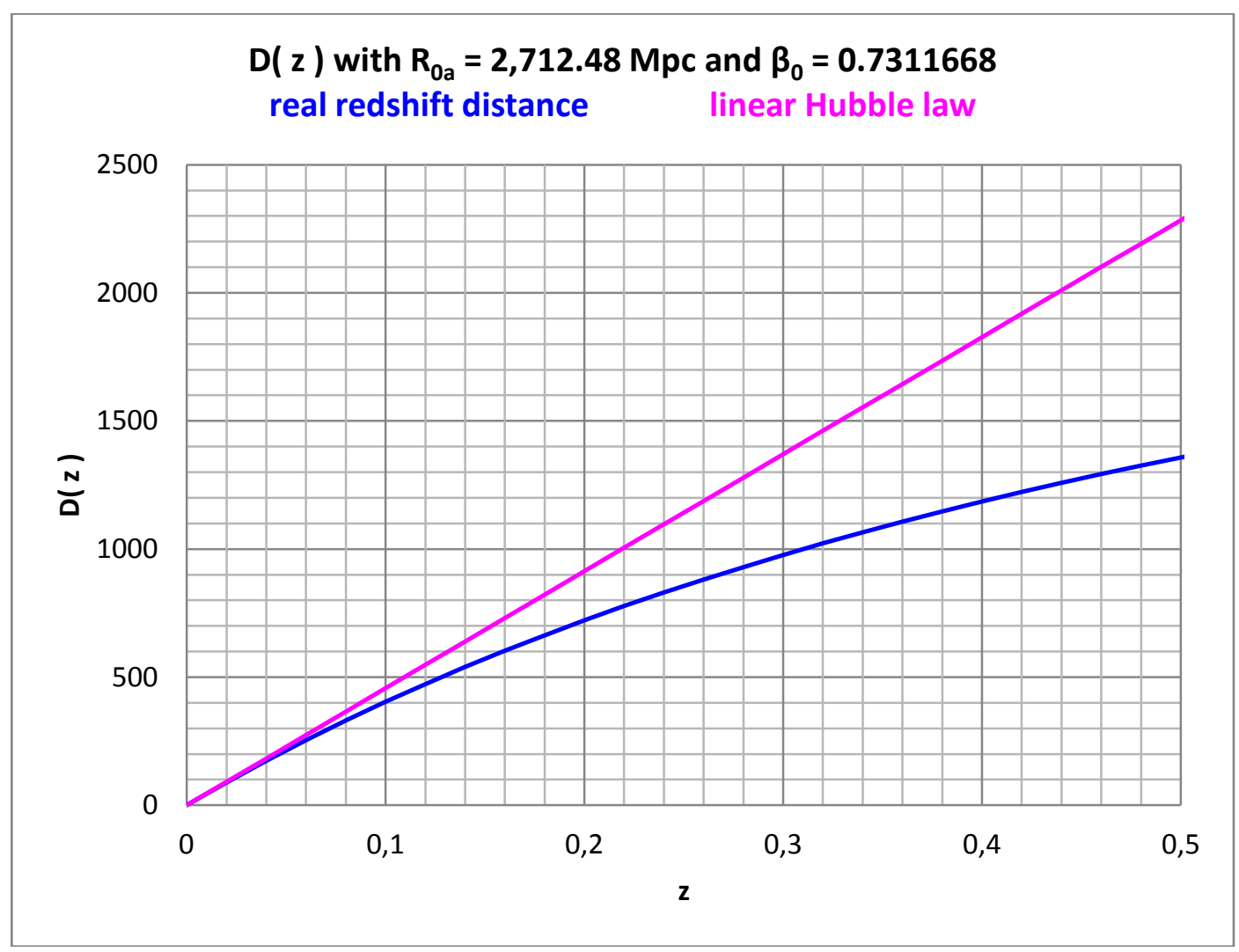

Figure 16. Non-approximated redshift distance D compared to the linear Hubble redshift distance.

It can be seen that the two curves already clearly separate from each other at $\mathrm{z} \approx 0.04$, and that Hubble's law results in distances that are significantly too large for larger redshifts, so that it is no longer applicable from around this value.

Recall:

Of course, it should be noted that the Hubble parameter $\mathrm{H}_{0 \mathrm{a}}$ in our theory results from an approximation for small redshifts $\mathrm{z}$. 


\subsection{Mean values}

If we replace in the equation (I, 38)

$$
m\left(z ; m_{0 a}, \beta_{0}\right)=5 \log _{10}\left[\frac{1}{\beta_{0}}\left(1-\frac{1}{\sqrt{1+z}}\right)+z\right]-5 \log _{10}(1+z)+m_{0 a}
$$

the parameter $\mathrm{m}_{0 \mathrm{a}}$ using equation $(\mathrm{I}, 47)$

$$
m_{0 a}=5 \log _{10}\left(R_{0 a}\right)-5+M \quad\left[R_{0 a}\right]=p c
$$

we get

$$
m\left(z ; R_{0 a}, \beta_{0}\right)=5 \log _{10}\left[\frac{1}{\beta_{0}}\left(1-\frac{1}{\sqrt{1+z}}\right)+z\right]-5 \log _{10}(1+z)+5 \log _{10}\left(R_{0 a}\right)-5+M
$$

From this equation it follows immediately

$$
\left.R_{0 a}\left(z, m ; M, \beta_{0}\right)=\frac{(1+z)}{\left[\frac{1}{\beta_{0}}\left(1-\frac{1}{\sqrt{1+z}}\right)+z\right]} 10^{\left[\frac{(m-M)}{5}+1\right.}\right]
$$

or, if we introduce the distance module $\mu=\mathrm{m}-\mathrm{M}$

$$
R_{0 a}\left(z ; \mu, \beta_{0}\right)=\frac{(1+z)}{\left[\frac{1}{\beta_{0}}\left(1-\frac{1}{\sqrt{1+z}}\right)+z\right]} 10^{\left(\frac{\mu}{5}+1\right)} \quad \text { mit } \quad \mu=m-M
$$

Note:

While the redshift $\mathrm{z}$ and the apparent magnitude $\mathrm{m}$ are actual measured variables, the distance module $\mu$ is to be regarded as a parameter because the absolute magnitude $\mathrm{M}$ cannot be measured directly.

The parameter $\beta_{0}$ is known to us from the evaluation of the Quasar catalog by Véron-Cetty [2]. In [4] the following parameters characterizing all 27 SNIa are given: absolute magnitude $\mathrm{M}_{\mathrm{B}}$, redshift $\mathrm{z}$ and maximum apparent magnitude $\mathrm{m}_{\mathrm{B}}$.

This allows us to calculate the associated $\mathrm{R}_{0 \mathrm{a}}$, ${ }_{\mathrm{i}}$ for all $\mathrm{SNIa}_{\mathrm{i}}$ (i numbers the individual SNIa). Table 6 shows the result:

\begin{tabular}{|c|c|c|c|c|c|}
\hline $\mathbf{z}_{\mathbf{i}}$ & $\mathbf{R}_{\mathbf{0 a}, \mathbf{i}}[\mathbf{M p c}]$ & $\mathbf{z}_{\mathbf{i}}$ & $\mathbf{R}_{\mathbf{0 a}, \mathbf{i}}[\mathbf{M p c}]$ & $\mathbf{z}_{\mathbf{i}}$ & $\mathbf{R}_{\mathbf{0 a}, \mathbf{i}}$ [ $\mathbf{M p c}$ ] \\
\hline 0.00435635 & $2,685.59$ & 0.00229826 & $2,989.52$ & 0.00845251 & $2,160.13$ \\
\hline 0.00350242 & $2,620.06$ & 0.00349242 & $3,130.03$ & 0.00456316 & $2,811.99$ \\
\hline 0.00435635 & $2,685.59$ & 0.00621763 & $3,059.04$ & 0.00151772 & $2,602.36$ \\
\hline 0.00229826 & $2,868.15$ & 0.00807892 & $2,850.38$ & 0.00435635 & $2,636.57$ \\
\hline 0.00350242 & $3,608.37$ & 0.00748518 & $2,242.44$ & 0.00350242 & $2,749.87$ \\
\hline 0.00350242 & $2,699.68$ & 0.00435635 & $2,685.59$ & 0.00434300 & $2,543.10$ \\
\hline 0.00517691 & $3,137.28$ & 0.00661458 & $2,079.37$ & 0.00482667 & $2,956.51$ \\
\hline 0.00629102 & $2,821.83$ & 0.00435635 & $2,636.57$ & 0.00470325 & $2,488.62$ \\
\hline
\end{tabular}




\begin{tabular}{|c|c|c|c|c|c|}
0.00456316 & $3,069.12$ & 0.00567726 & $2,088.18$ & 0.00423960 & $2,902.49$ \\
\hline$\left\langle\mathbf{R}_{0 \mathrm{a}}\right\rangle=$ & $2,733.65$ & \multicolumn{3}{|l}{} \\
\cline { 1 - 3 }
\end{tabular}

Table 6. Various distances $\mathrm{R}_{0 \mathrm{a}, \mathrm{i}}$ of the $27 \mathrm{SNIa}_{\mathrm{i}}$ calculated using the distance modules $\mu_{\mathrm{i}}$.

It may seem strange that we get a different value for $\mathrm{R}_{0 \mathrm{a}, \mathrm{i}}$ for each $\mathrm{SNIa}_{\mathrm{i}}$, which is actually the current physical distance of the observer from the coordinate origin $(r=0)$. In particular, the $R_{0 a, i}$ for almost equal redshifts $z_{i}$ should match!

But if we form the mean value of the 27 calculated values $\mathrm{R}_{0 \mathrm{a}, \mathrm{i}}$, we find $\left\langle\mathrm{R}_{0 \mathrm{a}}\right\rangle \approx 2,733.65 \mathrm{Mpc}$. This value is very close to the value $\mathrm{R}_{0 \mathrm{a}} \approx 2,712.48 \mathrm{Mpc}$, which we found above.

Overall, we must obvious conclude that the part of cosmology we are considering is essentially a science of averages.

In principle, this could be seen clearly from the beginning, if we retrospectively look a little more closely about the evaluation we carried out, e.g. the Quasar catalog and the subsequent finding of $\mathrm{R}_{0 \mathrm{a}}$.

Only the consideration of a large number of cosmic objects results in the correct values of astrophysically and cosmologically relevant quantities, respectively, which then are partly mean values only.

\section{Concluding remarks}

The light path $\mathrm{D}(\mathrm{z})$ of the photons through the expanding universe corresponds to a dynamic distance and is therefore an apparent one. This distance is not identical to the today's distance $\mathrm{D}_{0}(\mathrm{z})$ between the objects. For every conceivable observer, the cosmic objects are not spatially where they appear at first glance! In cosmology nothing is what it seems to be if we look at distances.

Of course, all cosmologically relevant astrophysical objects have a today's distance $\mathrm{D}_{0}$. However, this is not observable, but we can calculate it.

Photons that are emitted at this distance from the observed galaxy cannot have reached us so far.

A fundamental property of quantum mechanics is that it can only make probability statements about the microscopic objects it deals with. Here it is seen that both the measuring and the theorizing astrophysics and cosmology, respectively, strictly speaking, can only make statements about mean values of very distant and large objects.

This may be one of the reasons why both theories - the theory for the extremely small and the theory for the extremely large - do not fit together; i.e. cannot be brought together.

\section{Note of thanks:}

I would like to thank my wife for the long-standing toleration and the corresponding endurance of my almost constant virtual absence. What would I be without her?!

\section{Appendix}

In this table appendix, we provide the essential data that we have used and some of the data that we have edited or generated for general purposes.

\begin{tabular}{|c|c|c|c|c|c|c|c|c|}
\hline$\langle\mathbf{V}\rangle_{\mathbf{i}}$ & $\langle\mathbf{z}\rangle_{\mathbf{i}}$ & $\boldsymbol{\sigma}_{\mathrm{m}, \mathrm{i}}$ & $\langle\mathbf{V}\rangle_{\mathbf{i}}$ & $\langle\mathbf{z}\rangle_{\mathbf{i}}$ & $\boldsymbol{\sigma}_{\mathrm{m}, \mathrm{i}}$ & $\langle\mathbf{V}\rangle_{\mathbf{i}}$ & $\langle\mathbf{z}\rangle_{\mathbf{i}}$ & $\boldsymbol{\sigma}_{\mathrm{m}, \mathrm{i}}$ \\
\hline 17.12072194 & 0.269543711 & 1.25551062 & 19.5118161 & 1.28508799 & 0.79265674 & 19.7439932 & 1.86740102 & 0.8223715 \\
\hline 18.42994924 & 0.434725324 & 0.69496662 & 19.4960406 & 1.30997857 & 0.82617985 & 19.7431839 & 1.90379949 & 0.8745066 \\
\hline 18.77986464 & 0.514410603 & 0.68208433 & 19.5406994 & 1.33635871 & 0.79628275 & 19.73815 & 1.91629442 & 0.85608298 \\
\hline 18.92177101 & 0.571495206 & 0.70268585 & 19.5648675 & 1.36044896 & 0.84936023 & 19.7370051 & 1.94113536 & 0.83013271 \\
\hline 19.01993232 & 0.621120135 & 0.69571033 & 19.5526283 & 1.38646193 & 0.85285126 & 19.6390299 & 1.96661139 & 0.91303871 \\
\hline
\end{tabular}




\begin{tabular}{|c|c|c|c|c|c|c|c|c|}
\hline 19.07454597 & 0.665043993 & 0.72385254 & 19.5667343 & 1.41249746 & 0.82510058 & 19.7247377 & 1.99498872 & 0.83486627 \\
\hline 19.10685279 & 0.710045685 & 0.76943643 & 19.5917766 & 1.43823632 & 0.84883691 & 19.7073435 & 2.02761873 & 0.85770271 \\
\hline 19.20756345 & 0.750830795 & 0.74776464 & 19.5835759 & 1.46348111 & 0.81435344 & 19.7225437 & 2.05895826 & 0.83582282 \\
\hline 19.23878173 & 0.788362662 & 0.82397969 & 19.6146701 & 1.4877084 & 0.77561435 & 19.7209927 & 2.09067964 & 0.87548608 \\
\hline 19.34673999 & 0.823077834 & 0.84208852 & 19.6560914 & 1.50872984 & 0.80798031 & 19.7166723 & 2.12286464 & 0.87190043 \\
\hline 19.35605189 & 0.85711675 & 0.83026192 & 19.6421545 & 1.53039989 & 0.82193001 & 19.7562211 & 2.15726452 & 0.83914146 \\
\hline 19.35379019 & 0.889902425 & 0.83562264 & 19.6730062 & 1.55031021 & 0.78502817 & 19.6955838 & 2.1915251 & 0.87311109 \\
\hline 19.35354202 & 0.925268472 & 0.83309066 & 19.669718 & 1.57141117 & 0.81671189 & 19.7102256 & 2.23148844 & 0.89180926 \\
\hline 19.36111675 & 0.958962211 & 0.80795962 & 19.691489 & 1.59370615 & 0.79783244 & 19.6203328 & 2.27565595 & 0.8814518 \\
\hline 19.36687535 & 0.99085674 & 0.81407063 & 19.6689622 & 1.61663057 & 0.79869119 & 19.6516638 & 2.32895262 & 0.90747466 \\
\hline 19.39208122 & 1.021072758 & 0.83447413 & 19.7130344 & 1.64024196 & 0.79496734 & 19.7034969 & 2.39616356 & 0.89952989 \\
\hline 19.41216018 & 1.049862944 & 0.81581048 & 19.7208742 & 1.66227637 & 0.79948606 & 19.6915454 & 2.47184715 & 0.93743249 \\
\hline 19.43737733 & 1.076128596 & 0.81828949 & 19.7568415 & 1.68460462 & 0.79535961 & 19.7660462 & 2.57089058 & 0.97654953 \\
\hline 19.47736041 & 1.10186802 & 0.79353868 & 19.6973942 & 1.70912747 & 0.83259167 & 19.7708009 & 2.71401918 & 0.95905229 \\
\hline 19.4307727 & 1.129618161 & 0.80360659 & 19.7453187 & 1.7323057 & 0.83488167 & 19.7781162 & 2.90122279 & 0.85728912 \\
\hline 19.45345178 & 1.157690919 & 0.80262312 & 19.7723632 & 1.75403384 & 0.80160723 & 19.9208291 & 3.05796277 & 0.78948482 \\
\hline 19.4499718 & 1.18469656 & 0.81310891 & 19.7568754 & 1.77625888 & 0.80788436 & 20.0279357 & 3.20401523 & 0.77347127 \\
\hline 19.50609701 & 1.208890017 & 0.7810332 & 19.7599436 & 1.79742358 & 0.80969081 & 20.2283362 & 3.40521263 & 0.78550396 \\
\hline 19.48940778 & 1.233098139 & 0.80906834 & 19.7587704 & 1.82113988 & 0.83363286 & 20.5549521 & 3.7254264 & 0.73269653 \\
\hline 19.47597857 & 1.259028765 & 0.79685819 & 19.7435195 & 1.84394303 & 0.82211045 & 21.3169261 & 4.34427862 & 1.27303027 \\
\hline & & & & & & & & \\
\hline
\end{tabular}

Table 7. Mean values from the Quasar data set used according to [2].

$\langle\mathrm{z}\rangle_{\mathrm{i}}$ (with $\left.\mathrm{i}=1 \ldots 75\right)$ are the 75 mean values of the redshifts of the quasars in the redshift intervals formed. $\langle\mathrm{V}\rangle_{\mathrm{i}}$ are the associated 75 mean values of the apparent visual magnitude of the quasars.

$\sigma_{\mathrm{m}, \mathrm{i}}$ are the standard deviations with respect to the apparent magnitudes (m-axis in the redshift-magnitude diagram).

\begin{tabular}{|c|c|c|c|}
\hline $\mathbf{z}_{\mathbf{i}}$ (end of interval) & $\mathbf{N}_{\mathbf{i}}$ & $\mathbf{z}_{\mathbf{i}}$ (end of interval) & $\mathbf{N}_{\mathbf{i}}$ \\
\hline 0.24669 & 622 & 3.45369 & 128,884 \\
\hline 0.49338 & 3,891 & 3.70038 & 130,205 \\
\hline 0.74008 & 12,827 & 3.94708 & 131,357 \\
\hline 0.98677 & 25,495 & 4.19377 & 132,019 \\
\hline 1.23346 & 41,724 & 4.44046 & 132,432 \\
\hline 1.48015 & 58,818 & 4.68715 & 132,669 \\
\hline 1.72685 & 78,456 & 4.93385 & 132,848 \\
\hline 1.97354 & 97,109 & 5.18054 & 132,902 \\
\hline 2.22023 & 110,358 & 5.42723 & 132,924 \\
\hline 2.46692 & 117,810 & 5.67392 & 132,932 \\
\hline 2.71362 & 121,463 & 5.92062 & 132,949 \\
\hline 2.96031 & 123,820 & 6.16731 & 132,972 \\
\hline 3.20700 & 126,835 & 6.41400 & 132,977 \\
\hline
\end{tabular}

Table 8. Numbers $N_{i}$ summed up in the redshift intervals $Z_{i}$ of the quasars according to [2].

\begin{tabular}{|c|c|c|c|c|c|c|c|c|c|}
\hline SNIa & $\mu_{\text {TRGB }}$ & $\mu_{\mathrm{Ceph}}$ & $\boldsymbol{\mu}$ or $<\mu>$ & $\mathbf{m}_{\text {CSP_BO }}$ & $\mathbf{m}_{\mathrm{SC} \_\mathrm{B}}$ & $\mathbf{m}_{\mathrm{B}}$ or $\left\langle\mathrm{m}_{\mathrm{B}}\right\rangle$ & $\mathbf{M}_{\mathbf{i}}$ or $\left\langle\mathbf{M}_{\mathrm{i}}>\right.$ & $\mathbf{V}_{\text {NED }}$ & $\mathbf{z}$ \\
\hline $1980 \mathrm{~N}$ & 31.46 & 1 & 31.46 & 12.08 & & 12.08 & -19.38 & $1,306.00$ & 0.004356347 \\
\hline 1981B & 30.96 & 30.91 & 30.94 & 11.64 & 11.62 & 11.63 & -19.31 & $1,050.00$ & 0.003502423 \\
\hline 1981D & 31.46 & 1 & 31.46 & 11.99 & 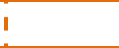 & 11.99 & -19.47 & $1,306.00$ & 0.004356347 \\
\hline 1989B & 30.22 & i & 30.22 & 11.16 & 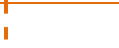 & 11.16 & -19.06 & 689.00 & 0.002298257 \\
\hline $1990 \mathrm{~N}$ & & 31.53 & 31.53 & 12.62 & 12.42 & 12.52 & -19.01 & $1,050.00$ & 0.003502423 \\
\hline 1994D & 31.00 & I & 31.00 & 11.76 & 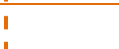 & 11.76 & -19.24 & $1,050.00$ & 0.003502423 \\
\hline
\end{tabular}




\begin{tabular}{|c|c|c|c|c|c|c|c|c|}
\hline $1994 \mathrm{ae}$ & 32.27 & 32.07 & $\quad 32.17$ & 12.94 & 12.92 & 12.93 & -19.24 & $1,552.00 \quad 0.005176915$ \\
\hline 1995al & 32.22 & 32.50 & 32.36 & 13.02 & 12.97 & 13.00 & -19.37 & \begin{tabular}{ll|l}
$1,886.00$ & 0.006291019 \\
\end{tabular} \\
\hline $1998 \mathrm{aq}$ & & 31.74 & 31.74 & 12.46 & 12.24 & 12.35 & -19.39 & $1,368.00 \quad 0.004563157$ \\
\hline 1998bu & 30.31 & & 30.31 & 11.01 & i & 11.01 & -19.30 & $689.00 \quad 0.002298257$ \\
\hline 2001el & 31.32 & 31.31 & 31.32 & 12.30 & 12.20 & 12.25 & -19.07 & $1,047.00: 0.003492416$ \\
\hline $2002 \mathrm{fk}$ & 32.50 & 32.52 & 32.51 & 13.33 & 13.20 & 13.27 & -19.25 & $1,864.00 \quad 0.006217635$ \\
\hline $2003 \mathrm{du}$ & & 32.92 & 32.92 & 13.47 & 13.47 & 13.47 & -19.45 & $2,422.00 \div 0.008078922$ \\
\hline $2005 \mathrm{cf}$ & & 32.26 & 32.26 & 12.96 & | 13.01 & 12.99 & -19.28 & $2,244.00 \quad 0.007485178$ \\
\hline 2006dd & 31.46 & $\begin{array}{ll}1 \\
1\end{array}$ & 31.46 & 12.38 & 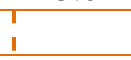 & 12.38 & -19.08 & $1,306.00 \quad 0.004356347$ \\
\hline 2007af & 31.82 & 31.79 & 31.81 & 12.72 & 12.70 & 12.71 & -19.10 & 1,983.00 0.006614576 \\
\hline 2007on & 31.42 & 1 & 31.42 & 12.39 & 1 & 12.39 & -19.03 & $1,306.00 \| 0.004356347$ \\
\hline $2007 \mathrm{sr}$ & 31.68 & 31.29 & 31.49 & 12.30 & 12.24 & 12.27 & -19.22 & $1,702.00: 0.005677261$ \\
\hline 2009ig & & 32.50 & 32.50 & 13.29 & 13.46 & 13.38 & -19.13 & $2,534.00 \quad 0.008452514$ \\
\hline 2011by & & 31.59 & 31.59 & 12.63 & 12.49 & 12.56 & -19.03 & $1,368.00 \quad 0.004563157$ \\
\hline $2011 \mathrm{fe}$ & 29.08 & 29.14 & 29.11 & 9.82 & 9.75 & 9.79 & -19.33 & $455.00 \quad 0.001517717$ \\
\hline $2011 \mathrm{iv}$ & 31.42 & $\begin{array}{l}1 \\
1\end{array}$ & 31.42 & 12.03 & 1 & 12.03 & -19.39 & $1,306.00 \quad 0.004356347$ \\
\hline $2012 \mathrm{cg}$ & 31.00 & 31.08 & 31.04 & 11.72 & 11.55 & 11.64 & -19.41 & $1,050.00 \quad 0.003502423$ \\
\hline $2012 \mathrm{fr}$ & 31.36 & 31.31 & 31.34 & 12.09 & 11.92 & 12.01 & -19.33 & $1,302.00 \div 0.004343005$ \\
\hline 2012ht & & 31.91 & 31.91 & 12.66 & 12.70 & 12.68 & -19.23 & $1,447.00 \quad 0.004826672$ \\
\hline $2013 d y$ & & 31.50 & 31.50 & 12.23 & 12.31 & 12.27 & -19.23 & $1,410.00 \quad 0.004703254$ \\
\hline $2015 \mathrm{~F}$ & & 31.51 & 31.51 & 12.40 & 12.28 & 12.34 & -19.17 & $1,271.00 \quad 0.0042396$ \\
\hline & & & & & & $\langle\mathbf{M}\rangle=$ & -19.24 & \\
\hline
\end{tabular}

Table 9. Summary of the data which we used from the 27 SNIa according to [4].

SNIa values that can be traced back to a mean value are marked in green (bold).

The individual meanings of the data can be found in the article mentioned.

The data for the angular-size redshift diagram can be found in full in [3].

\section{References}

[1] Haase, S. New Derivation of Redshift Distance without Using Power Expansions. Preprints 2021, 2021120039 (doi: 10.20944/preprints202112.0039.v1).

[2] M.-P. Véron-Cetty and P. Véron, A Catalogue of Quasars and Active Nuclei, $13^{\text {th }}$ edition, March 2010, http://www.obs-hp.fr/catalogues/veron2_13/veron2_13.html

[3] K. Nilsson, M. J. Valtonen, J. Kotilainen and T. Jaakkola, Astro. J. 413 (1993), 453.

[4] W. L. Freedman u. a., The Carnegie-Chicago Hubble Program. VIII. An Independent Determination of the Hubble Constant Based on the Tip of the Red Giant Branch, arXiv.org:1907.05922

[5] The Planck Collaboration: Planck 2018 results. VI. Cosmological parameters, arXiv:1807.06209

[6] The Event Horizon Telescope Collaboration, The Astrophysical Journal Letters, 875:L1 (17pp), 2019 April 10, https://doi.org/10.3847/2041-8213/ab0ec7

[7] de.wikipedia.org/wiki/Messier_87, abgerufen am 18.12.2021

[8] de.wikipedia.org/wiki/Quasar, abgerufen am 18.12.2021

[9] de.wikipedia.org/wiki/UDFj-39546284, abgerufen am 18.12.2021

[10] G. Dautcourt, Was sind Quasare?, S. 68, Abb. 18, BSB B.G. Teubner Verlagsgesellschaft, 4. Auflage 1987 


\section{Copyright:}

This text is subject to German and international copyright law, i.e. the publication, translation, transfer to other media, etc. - including parts - is permitted only with the prior permission of the author.

Copyright by Steffen Haase, Germany, Leipzig, 2022 